\title{
Numerical Simulation of Solar Cells and Solar Cell Characterization Methods: the Open-Source on Demand Program AFORS-HET
}

\author{
Rolf Stangl, Caspar Leendertz and Jan Haschke \\ Helmholtz-Zentrum Berlin für Materialien und Energie, \\ Institut für Silizium Photovoltaik, Kekule-Str.5, D-12489 Berlin
}

Germany

\section{Introduction}

Within this chapter, the principles of numerical solar cell simulation are described, using AFORS-HET (automat for simulation of heterostructures). AFORS-HET is a one dimensional numerical computer program for modelling multi layer homo- or heterojunction solar cells as well as some common solar cell characterization methods.

Solar cell simulation subdivides into two parts: optical and electrical simulation. By optical simulation the local generation rate $G(\mathbf{x}, t)$ within the solar cell is calculated, that is the number of excess carriers (electrons and holes) that are created per second and per unit volume at the time $t$ at the position $\mathbf{x}$ within the solar cell due to light absorption. Depending on the optical model chosen for the simulation, effects like external or internal reflections, coherent superposition of the propagating light or light scattering at internal surfaces can be considered. By electrical simulation the local electron and hole particle densities $n(\mathbf{x}, t), p(\mathbf{x}, t)$ and the local electric potential $\varphi(\mathbf{x}, t)$ within the solar cell are calculated, while the solar cell is operated under a specified condition (for example operated under open-circuit conditions or at a specified external cell voltage). From that, all other internal cell quantities, such like band diagrams, local recombination rates, local cell currents and local phase shifts can be calculated. In order to perform an electrical simulation, (1) the local generation rate $G(\mathbf{x}, t)$ has to be specified, that is, an optical simulation has to be done, (2) the local recombination rate $R(\mathbf{x}, t)$ has to be explicitly stated in terms of the unknown variables $n, p, \varphi, R(\mathbf{x}, t)=f(n, p, \varphi)$. This is a recombination model has to be chosen. Depending on the recombination model chosen for the simulation, effects like direct band to band recombination (radiative recombination), indirect band to band recombination (Auger recombination) or recombination via defects (Shockley-Read-Hall recombination, dangling-bond recombination) can be considered.

In order to simulate a real measurement, the optical and electrical simulations are repeatedly calculated while changing a boundary condition of the problem, which is specific to the measurement. For example, the simulation of a $\mathrm{i}-\mathrm{V}$ characteristic of a solar cell is done by calculating the internal electron and hole current (the sum of which is the total current) as a function of the externally applied voltage.

Source: Solar Energy, Book edited by: Radu D. Rugescu,

ISBN 978-953-307-052-0, pp. 432, February 2010, INTECH, Croatia, downloaded from SCIYO.COM 
Most solar cells, which are on the market today, can be described as a one dimensional sequence of different semiconductor layers. If they are uniformly illuminated, a one dimensional solar cell modelling is sufficient (the internal electron/hole current can flow only in one direction). This is the case for most wafer based silicon solar cells as well as for most thin film solar cells on glass as long as the integrated series connection shall not be explicitly modelled, see Fig.1 (left).
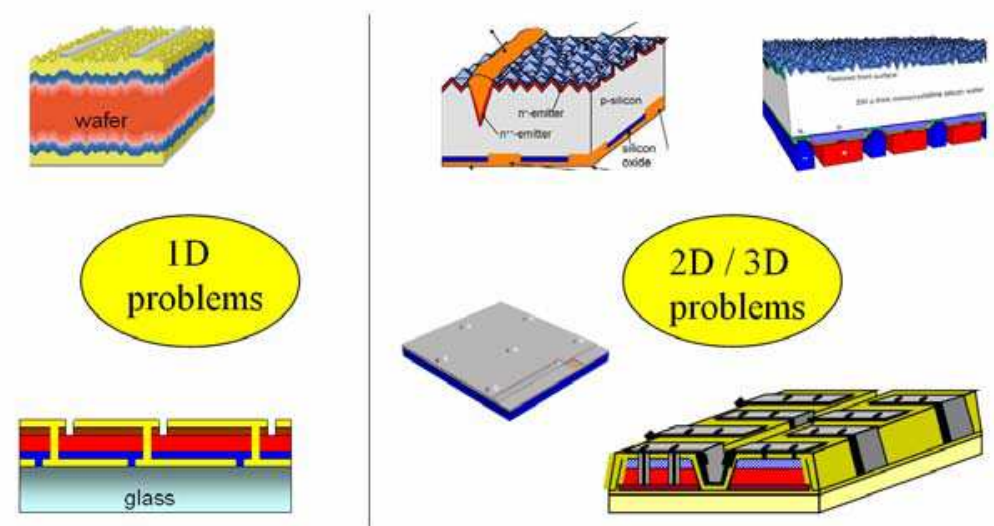

Fig. 1. solar cell structures which can be treated as a one dimensional problem (left), or which have to be treated as a two or even three dimensional problem (right).

However, in order to minimize contact recombination, stripe- or point-like metallic contacts which are embedded within an insulating passivation layer (i.e. silicon nitride, silicon oxide) are sometimes introduced. These contacts can either be placed on both sides of the solar cell or favourably only at the rear side of the solar cell, thereby avoiding shadowing due to the contacts. In these cases, the resulting solar cells have to be modelled as two or even three dimensional problems (the internal electron/hole current can flow in 2 or even 3 directions), see Fig.1 (right). In the current version 2.4 of AFORS-HET only 1D simulations are possible; however, there is a 2D mode under development.

Another possibility to reduce contact recombination is the use of heterojunctions, that is different semiconductors are used to form the solar cell absorber (photon collecting area), the electron extracting area and the hole extracting area of the solar cell. Ideally, the excess carriers of the solar cell absorber (electrons and holes) should be selectively attracted/repelled towards the contacts, see Fig. 2. These selective contacts can be either conventionally realized by doping/counter doping of the solar cell absorber, leading to a formation of an internal electric field by which the selective excess carrier separation is achieved. In this case, homojunctions will form, i.e. there are no band offsets, as the absorber and the electron/hole extracting areas of the solar cell consist of the same semiconductor. In principle, if different semiconductors with appropriately matched work functions are used to form the electron/hole extracting areas, heterojunctions can be formed having the same internal electric field as the homojunction, but with additional band offsets that enhance the repelling character of the contacts, see Fig. 2 (right).

A heterojunction solar cell will thus have a higher open circuit voltage compared to a homojunction solar cell. Less excess carriers of the repelled type are transported into the 


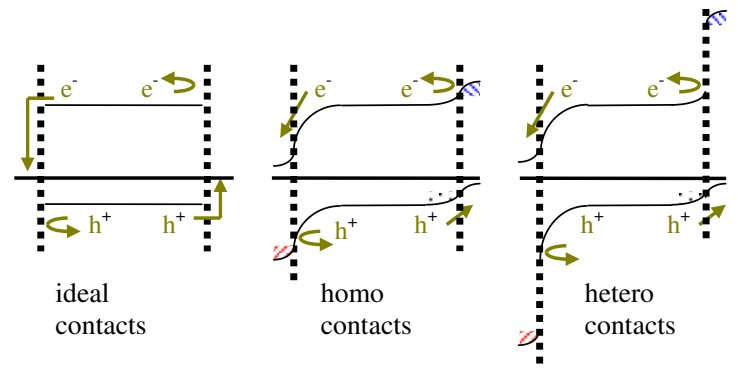

Fig. 2. schematic sketch of selective absorber contacts (band diagrams of a p-type semiconductor used as an absorber material). Ideal contacts (left), homojunction contacts (middle) and ideally aligned heterojunction contacts (right). NOTE: The dimensions of the $x$ axis are schematic and not in scale!

electron/hole collecting regions, and thus the contact recombination at the metallic contacts is reduced. However, an essential pre-requisite is not to create too many interface defects during the formation of the heterojunction at the interface between the absorber and the electron/hole collecting area, which will otherwise act as additional recombination centres. A realistic computer program for solar cell modelling should therefore be able to handle homojunctions as well as heterojunctions, and it should be able to consider interface defects and the corresponding interface recombination $R^{i t}(t)$. Depending on the physical assumption how to describe an electron/hole transport across a heterojunction interface, a distinct interface model has to be chosen. For example, within the current version of AFORS-HET 2.4 a drift-diffusion and a thermionic emission interface model can be chosen, allowing the placement of interface defects but neglecting tunnelling. Tunneling interface models are under development.

To assure a numerical simulation with reliable results, a good model calibration, i.e. a comparison of simulation results to a variety of different characterisation methods, is necessary. The solar cell under different operation conditions should be compared to the simulations. Also different characterisation methods for the solar cell components, i.e. for the individual semiconductor layers and for any sub stacks should be tested against simulation. Only then the adequate physical models as well as the corresponding model input parameters can be satisfactory chosen. Thus a good solar cell simulation program should be able to simulate the common characterisation methods for solar cells and its components.

In this chapter, we describe AFORS-HET (automat for simulation of heterostructures), a one dimensional numerical computer program to simulate solar cells as well as typical solar cell characterisation methods. Thus a variety of different measurements on solar cell components or on the whole solar cell can be compared to the corresponding simulated measurements in order to calibrate the parameters used in the simulations.

All optical and electrical models, which can be used in AFORS-HET, are discussed and their mathematical and physical background is stated. Furthermore, many solar cell characterisation methods, which can be simulated by AFORS-HET, are sketched. The difference in modelling thick film (wafer based) or thin film solar cells on glass will be investigated in order to choose the appropriate model. The basic input parameters of the corresponding models are described. Some selected results in modelling wafer based amorphous/crystalline silicon solar cells illustrate the concepts of numerical solar cell simulation within practical applications. 


\section{Brief description of AFORS-HET}

The current version 2.4 of AFORS-HET solves the one dimensional semiconductor equations (Poisson's equation and the transport and continuity equation for electrons and holes) with the help of finite differences under different conditions, i.e.: (a) equilibrium mode (b) steady state mode, (c) steady state mode with small additional sinusoidal perturbations, (d) simple transient mode, that is switching external quantities instantaneously on/off, (e) general transient mode, that is allowing for an arbitrary change of external quantities. A multitude of different physical models has been implemented. The generation of electron/hole pairs (optical models of AFORS-HET) can be described either by Lambert-Beer absorption including rough surfaces and using measured reflection and transmission files, or by calculating the plain surface incoherent/coherent multiple internal reflections, using the complex indices of reflection for the individual layers. Different recombination models can be considered within AFORS-HET: radiative recombination, Auger recombination, Shockley-Read-Hall and/or dangling-bond recombination with arbitrarily distributed defect states within the bandgap. Super-bandgap as well as sub-bandgap generation/recombination can be treated. The following interface models for treating heterojunctions are implemented: Interface currents can be modelled to be either driven by drift diffusion or by thermionic emission. A band to trap tunnelling contribution across a hetero-interface can be considered. The following boundary models can be chosen: The metallic contacts can be modelled as flatband or Schottky like metal/semiconductor contacts, or as metal/insulator/semiconductor contacts. Furthermore, insulating boundary contacts can also be chosen.

Thus, all internal cell quantities, such as band diagrams, quasi Fermi energies, local generation/recombination rates, carrier densities, cell currents and phase shifts can be calculated. Furthermore, a variety of solar cell characterisation methods can be simulated, i.e.: current voltage, quantum efficiency, transient or quasi-steady-state photo conductance, transient or quasi-steady-state surface photovoltage, spectral resolved steady-state or transient photo- and electro-luminescence, impedance/admittance, capacitance-voltage, capacitancetemperature and capacitance-frequency spectroscopy and electrical detected magnetic resonance. The program allows for arbitrary parameter variations and multidimensional parameter fitting in order to match simulated measurements to real measurements.

AFORS-HET, version 2.4, is an open source on demand program. If you want to contribute send an e-mail to AFORS-HET@helmholtz-berlin.de, specifying in detail what you would like to implement. It is distributed free of charge and it can be downloaded via internet:

http://www.helmholtz-berlin.de/forschung/enma/si-pv/projekte/asicsi/afors-het/index_en.html

\section{Basic input parameter of AFORS-HET and associated physical models}

\subsection{Optical parameter (super bandgap generation optical models)}

The incoming spectral photon flux $\Phi_{0}(\lambda, t)$, that is the number of incident photons of wavelength $\lambda$ at the time $t$, has to be stated. In order to calculate the local super-bandgap generation rate $G(x, t)$ within the semiconductor stack, that is the number electrons and holes that are created per second and per unit volume at the time $t$ at the position $x$ due to super-bandgap light absorption, there are two optical models available: (1) Lambert-Beer absorption and (2) coherent/incoherent internal multiple reflections. For both models, the thicknesses $L_{i}$ and the dielectric properties of the semiconductor layers have to be specified, 
i.e. the complex refractive indices, $\tilde{n}_{i}(\lambda)=n_{i}(\lambda)-\mathrm{i} k_{i}(\lambda)$ with refractive index $n(\lambda)$ and extinction coefficient $k(\lambda)$. If the model Lambert-Beer absorption is chosen, a measured reflectivity $R(\lambda)$ of the semiconductor stack can be specified, and the resulting absorption $A(\lambda, x, t)$ within the semiconductor stack will be calculated, assuming Lambert Beer absorption by using the specified values for $k_{i}(\lambda)$ only and performing a ray tracing in order to account for textured surfaces and multiple bouncing of the radiation within the stack. If the model coherent/incoherent internal multiple reflections is chosen, the reflectivity $R(\lambda)$, the transmisivity $T(\lambda)$ and the absorption $A(\lambda, x, t)$ of the semiconductor stack is calculated from the specified values $n_{i}(\lambda), k_{i}(\lambda)$, assuming plain surfaces within the stack but taking coherent internal multiple reflections into account, if desired. For both models, $G(x, t)$ is calculated from $A(\lambda, x, t)$ by integration over all wavelengths of the incident spectrum. In order to model optical sub-bandgap generation, optical electron/hole capture cross sections $\sigma_{n, \text { opt }} \neq 0, \sigma_{p, o p t} \neq 0$ for the Shockley-Read-Hall defects have to be specified.

\subsection{Layer parameter (semiconductor bulk models)}

For each semiconductor layer, the thickness $\mathrm{L}$, the electron/hole mobilities $\mu_{n}, \mu_{p}$, the effective valence/conduction band densities $N_{V}, N_{C}$, the electron/hole thermal velocities $v_{n}, v_{p}$, the electron affinity $\chi$, the relative dielectric constant $\varepsilon$, the doping profile $N_{D}(x)$, $N_{A}(x)$ and the bandgap $E_{g}$ of the semiconductor has to be specified. In order to describe recombination within the semiconductor, up to four different recombination models can be chosen, (1) radiative recombination, (2) Auger recombination, (3) Shockley-Read-Hall recombination, (4) dangling bond recombination. For radiative recombination, the radiative band to band rate constant $r^{b b}$ has to be specified (Sze \& Kwok, 2007). For Auger recombination, the electron/hole Auger rate constants $r_{n}^{\text {Aug }}, r_{p}^{\text {Aug }}$ have to be specified (Sze \& Kwok, 2007). For Shockley-Read-Hall recombination, the defect density distribution within the bandgap of the semiconductor $N_{\text {trap }}(E)$ and two capture cross sections $\sigma_{n}, \sigma_{p}$ and if needed also two optical capture cross sections $\sigma_{n}^{\text {opt }}, \sigma_{p}^{\text {opt }}$ for the electron/hole capture have to be specified (Sze \& Kwok, 2007). For dangling bond recombination, the defect distribution within the bandgap of the semiconductor $N_{\text {trap }}(E)$, four capture cross sections $\sigma_{n}^{+}, \sigma_{p}^{0}, \sigma_{n}^{0}, \sigma_{p}^{-}$and the correlation energy $U$ have to be specified (Sah \& Shockley, 1958). Optical capture is not yet implemented in case of dangling bond recombination.

\subsection{Interface parameter (semiconductor/semiconductor interface models)}

The electron/hole current transport across a semiconductor/semiconductor interface can be described by three different interface models, (1) no interface, (2) drift diffusion interface, (3) thermionic emission interface. If no interface is chosen, no additional interface defects can be specified. Otherwise, an interface defect distribution $N_{\text {trap }}^{i t}(E)$ can be specified. If the drift diffusion interface is chosen, an interface thickness $L_{i t}$ and interface capture cross sections $\sigma_{n}^{i t}, \sigma_{p}^{i t}$ have to be specified. For both models (1) and (2), transport across the semiconductor/semiconductor interface is treated according to the drift-diffusion approximation like in the bulk of the semiconductor layers (Sze \& Kwok, 2007). If the thermionic emission interface is chosen, the interface is regarded to be infinitively thin and 
four capture cross sections $\sigma_{n, I}^{i t}, \sigma_{n, I I}^{i t}, \sigma_{p, I}^{i t}, \sigma_{p, I I}^{i t}$ and if needed also four optical capture cross sections $\sigma_{n, I}^{i t, o p t}, \sigma_{n, I I}^{i t, o p t}, \sigma_{p, I}^{i t, o p t}, \sigma_{p, I I}^{i t, o p t}$ for electron/hole capture from both sides of the interface have to be specified. Transport across the interface is then treated according to the theory of thermionic emission (Sze \& Kwok, 2007).

\subsection{Boundary parameter (back/front contact to semiconductor boundary models)}

The boundaries of the semiconductor stack may either be metallic (usually constituting the contacts of the solar cell) or they may be insulating in order to simulate some specific measurements requiring insulator contacts. Four different boundary models can be chosen: (1) flatband metal/semiconductor contact, (2) Schottky metal/semiconductor contact, (3) insulator contact, (4) metal/insulator/semiconductor contact. If choosing the flatband metal/semiconductor contact, there will be no band banding induced within the semiconductor due to the contact (flatband contact). The electron/hole surface recombination velocities $S_{n}^{\text {front lback }}, S_{p}^{\text {front } / b a c k}$ of the metallic contact have to be specified (Sze \& Kwok, 2007). If choosing the Schottky metal/semiconductor contact, an additional work function $\phi^{\text {front } / \text { back }}$ of the metal contact has to be specified. A depletion or accumulation layer within the semiconductor due to the contact will then form according to Schottky theory (Sze \& Kwok, 2007). If choosing the insulator/semiconductor or the metal/insulator/semiconductor contact, interface states between the insulator and the semiconductor can be stated, that is an interface defect distribution $N_{\text {trap }}^{i t}(E)$ and interface capture cross sections $\sigma_{n}^{i t}, \sigma_{p}^{i t}$ have to be specified (Kronik \& Shapira, 1999). In case of the metal/insulator/semiconductor contact an additional interface capacity $C^{\text {front/back }}$ has to be specified (Kronik \& Shapira, 1999). Due to the interface defects a band bending within the semiconductor can form.

\subsection{Circuit elements}

A series resistance $R_{s}$, a parallel resistance $R_{p}$, a parallel capacitance $C_{p}$ and in case of an metal/insulator/semiconductor contact also a series capacitance $C_{s}$ can be specified. If circuit elements are specified, the internal cell voltage $V_{\text {int }}$ and the internal cell current $I_{\text {int }}$ of the semiconductor stack will differ from the external cell voltage $V_{e x t}$ and external cell current $I_{e x t}$ of the modeled device.

\subsection{External parameters}

External parameters are defined to be parameters which are externally applied to the device under consideration and which can also be easily varied in a real experiment. These are the temperature $T$ of the device, a spectral and a monochromatic illumination source leading to the spectral photon flux $\Phi_{0}(\lambda, t)$ required for the optical simulations, and the external cell voltage $V_{\text {ext }}(t)$ or the external cell current $I_{\text {ext }}(t)$ which is applied to the device. The remaining quantity, i.e. the external cell current $I_{e x t}(t)$ or the external cell voltage $V_{\text {ext }}(t)$ respectively, will be calculated.

\section{Mathematical description of the DGL system solved by AFORS-HET}

In the following, the differential equations and corresponding boundary conditions, which are solved by AFORS-HET under the various conditions, are stated. 
An arbitrary stack of semiconductor layers can be modeled. Within each semiconductor layer the Poisson equation and the transport and continuity equations for electrons and holes have to be solved. At each semiconductor/semiconductor interface and at the front and back side boundary of the stack the current transport through these interfaces/boundaries can be described by different physical models. It results a highly nonlinear coupled system of three differential equations with respect to time and space derivatives. The electron density $n(x, t)$, the hole density $p(x, t)$, and the electric potential $\varphi(x, t)$ are the independent variables, for which this system of differential equations is solved. It is solved according to the numerical discretisation scheme as outlined by Selberherr (Selberherr, 1984) in order to linearize the problem and using the linear SparLin solver which is available in the internet (Kundert et. al., 1988).

It can be solved for different calculation modes: (1) EQ calculation mode, describing thermodynamic equilibrium at a given temperature, (2) DC calculation mode, describing steady-state conditions under an external applied voltage or current and/or illumination, (3) AC calculation mode, describing small additional sinusoidal modulations of the external applied voltage/illumination, and (4) TR calculation mode, describing transient changes of the system, due to general time dependent changes of the external applied voltage or current and/or illumination.

In case of using the EQ or the DC calculation mode, all time derivatives vanish, resulting in a simplified system of differential equations. The system of differential equations is then solved for the time independent, but position dependent functions, $n^{E Q / D C}(x), p^{E Q / D C}(x)$, $\varphi^{E Q / D C}(x)$.

$$
\begin{array}{ll}
n(x, t)=n^{E Q}(x), & n(x, t)=n^{D C}(x) \\
p(x, t)=p^{E Q}(x) & p(x, t)=p^{D C}(x) \\
\varphi(x, t)=\varphi^{E Q}(x) & \varphi(x, t)=\varphi^{D C}(x)
\end{array}
$$

In case of using the AC calculation mode, it is assumed that all time dependencies can be described by small additional sinusoidal modulations of the steady-state solutions. All time dependent quantities are then modelled with complex numbers (marked by a dash $\sim$ ), which allows to determine the amplitudes and the phase shifts between them. I.e., for the independent variables of the system of differential equations, one gets:

$$
\begin{aligned}
& n(x, t)=n^{D C}(x)+\tilde{n}^{A C}(x) e^{i \omega t} \\
& p(x, t)=p^{D C}(x)+\tilde{p}^{A C}(x) e^{i \omega t} \\
& \varphi(x, t)=\varphi^{D C}(x)+\tilde{\varphi}^{A C}(x) e^{i \omega t}
\end{aligned}
$$

In case of using the TR calculation mode, the description of the system starts with a steadystate (DC-mode) simulation, specifying an external applied voltage or current and/or illumination. An arbitrary evolution in time of the external applied voltage or current and/or illumination can then be specified by loading an appropriate file. Then, the time evolution of the system, i.e. the functions $n(x, t), p(x, t), \varphi(x, t)$ during and after the externally applied changes are calculated. 


\subsection{Optical calculation: super bandgap generation models}

In order to describe the generation rate $G_{n}(x, t), G_{p}(x, t)$ of electrons and holes due to photon absorption within the bulk of the semiconductor layers, a distinction between superbandgap generation (for photons with an energy $E_{\text {photon }}=h c / \lambda \geq E_{g}$ ) and sub-bandgap generation (for photons with an energy $\left.E_{\text {photon }}=h c / \lambda \leq E_{g}\right)$ is made ( $\lambda$ : photon wavelength $h$ : Planck's constant, $c$ : velocity of light, $E_{g}$ : bandgap of the semiconductor layer in which the photon absorption takes place). Only the super-bandgap generation rate is calculated by optical modelling as it is independent of the local particle densities $n(x, t), p(x, t)$. Subbandgap generation depends on the local particle densities and must therefore be calculated within the electrical modeling part.

The optical super-bandgap generation rate is equal for electrons and holes $G(x, t)=G_{n}(x, t)=G_{p}(x, t)$. It can either be imported by loading an appropriate file (using external programs for its calculation) or it can be calculated within AFORS-HET.

So far, two optical models are implemented in AFORS-HET, i.e. the optical model LambertBeer absorption and the optical model coherent/incoherent internal multiple reflections. The first one takes textured surfaces and multiple internal boundary reflections into account (due to simple geometrical optics) but neglects coherence effects. It is especially suited to treat wafer based crystalline silicon solar cells. The second takes coherence effects into account, but this is done only for plain surfaces. If coherence effects in thin film solar cells are observable it may be used.

\subsubsection{Optical model: Lambert-Beer absorption}

Using this model, the absorption within the semiconductor stack will be calculated assuming simple Lambert-Beer absorption, allowing for multiple for and backward traveling of the incoming light, however disregarding coherent interference. A (measured) reflectance and absorptance file of the illuminated contact $R(\lambda), A(\lambda)$ can be loaded or constant values can be used. The incoming spectral photon flux $\Phi_{0}(\lambda, t)$ is weighted with the contact reflection and absorption, i.e. the photon flux impinging on the first semiconductor layer is given by $\Phi_{0}(\lambda, t) R(\lambda) A(\lambda)$. To simulate the extended path length caused by a textured surface, the angle of incidence $\delta$ of the incoming light can be adjusted. On a textured $\mathrm{Si}$ wafer with $<111>$ pyramids, this angle is $\delta=54.74^{\circ}$, whereas $\delta=0^{\circ}$ equals normal incidence. The angle $\gamma$ in which the light travels through the layer stack depends on the wavelength of the incoming light and is calculated according to Snellius' law:

$$
\gamma(\lambda)=\delta-\arcsin \left\{\sin (\delta) \cdot \frac{1}{n(\lambda)}\right\},
$$

whereas $n(\lambda)$ is the wavelength dependent refraction index of the first semiconductor layer at the illuminated side. Note, that within this model, the change in $\gamma(\lambda)$ is neglected, when the light passes a semiconductor/semiconductor layer interface with two different refraction indices. Thus it is assumed that all photons with a specified wavelength cross the layer stack under a distinct angle $\gamma$.

Photon absorption is then calculated from the spectral absorption coefficient $\alpha_{x}(\lambda)=4 \pi k(\lambda) / \lambda$ of the semiconductor layer corresponding to the position $\mathrm{x}$ within the stack, which is calculated from the provided extinction coefficient $k(\lambda)$ of the layer. The 
super bandgap electron/hole generation rate for one single run trough the layer stack (no multiple passes) is then given by:

$$
G(x, t)=\int_{\lambda_{\min }}^{\lambda_{\max }} d \lambda \Phi_{0}(\lambda, t) R(\lambda) A(\lambda) \alpha_{x}(\lambda) e^{\frac{-\alpha_{x}(\lambda) x}{\cos (\gamma)}} .
$$

The minimum and maximum wavelengths $\lambda_{\min }, \lambda_{\max }$ for the integration are generally provided by the loaded spectral range of the incoming spectral photon flux, $\Phi_{0}(\lambda, t)$. However, if necessary, $\lambda_{\max }$ is modified in order to ensure that only super-bandgap generation is considered: $\lambda_{\max } \leq h c / E_{g}$.

To simulate the influence of light trapping mechanisms, internal reflections at both contacts can be additionally specified. They can either be set as a constant value or wavelength dependant (a measured or calculated file can be loaded). The light then passes through the layer stack several times as defined by the user, thereby enhancing the absorptivity of the layer stack (the local generation rate). The residual flux after the defined number of passes is added to the transmitted flux at the contact, at which the calculation ended (illuminated or not-illuminated contact), disregarding the internal reflection definitions at this contact.

This model was designed to estimate the influence of light trapping of crystalline silicon solar cells and to adapt the simulation to real measurements. However, it neglects the internal multiple reflections and refractions within the layer stack.

\subsubsection{Optical model: coherent/incoherent internal multiple reflections}

Using this model, the absorption within the semiconductor stack will be calculated by modelling coherent or incoherent internal multiple reflections within the semiconductor stack. Additional non-conducting optical layers in front of the front contact/behind the back contact of the solar cell can be assumed, for example in order to model the effect of antireflection coatings. Normal incidence of the incoming illumination is assumed.

The reflectance, transmittance and absorptance of all layers (optical layers and the semiconductor layers) is calculated, using the concepts of complex Fresnel amplitudes. Each layer can be specified to be optically coherent or optically incoherent for a particular light beam (incident illumination). A layer is considered to be coherent if its thickness is smaller than the coherence length of the light beam that is incident on the system.

In order to be able to consider coherent effects, the specified incoming illumination $\Phi_{0}(\lambda, t)$ is modeled by an incoming electromagnetic wave, with a complex electric field component $\tilde{E}_{0}^{+}(\lambda, t)$ (front side illumination, electromagnetic wave traveling in positive direction towards the back contact, with $\Phi_{0}(\lambda, t)=$ Const $\left|\tilde{E}_{0}^{+}(\lambda, t)\right|^{2}$ ), or $\tilde{E}_{N+1}^{-}(\lambda, t)$ respectively (back side illumination, electromagnetic wave traveling in negative direction towards the front contact, with $\Phi_{0}(\lambda, t)=$ Const $\left.\left|\tilde{E}_{N+1}^{-}(\lambda, t)\right|^{2}\right)$. The complex electric field components of the travelling wave are raytraced according to the Fresnel formulas, and thus the resulting electromagnetic wave $\widetilde{E}(x, \lambda, t)$ at any position $\mathrm{x}$ within the layer stack is calculated. An incoherent layer is modeled by a coherent calculation of several electromagnetic waves within that layer (specified by the integer $N_{\text {incoherentIterations }}$ ), assuming some phase shift between them, and averaging over the resulting electric field components. 


\subsection{Electrical calculation - bulk layers: semiconductor bulk models}

Within the bulk of each semiconductor layer, Poisson's equation and the transport equations for electrons and holes are to be solved in one dimension. So far, there are two semiconductor bulk models available, i.e. the bulk model "standard semiconductor" and the bulk model "crystalline silicon". If using the standard semiconductor model, all bulk layer input parameters as specified in Chapter 3.2 can be individually adjusted. If using the crystalline silicon bulk model, most input parameters for crystalline silicon are calculated from few remaining input parameters, i.e. from the doping and defect densities $N_{D}(x)$, $N_{A}(x), N_{\text {trap }}$ of crystalline silicon. Thus effects like band gap narrowing or the doping dependence of the mobility or of the Auger recombination of crystalline silicon are explicitly modeled.

Within each layer, a functional dependence in space can be specified for the doping densities $N_{D}(x), N_{A}(x)$. These input parameters can be chosen to be (1) constant, (2) linear, (3) exponential, (4) Gaussian like, (5) error function like decreasing or increasing as a function of the space coordinate $x$.

\subsubsection{Bulk model: standard semiconductor}

The doping densities $N_{D}(x), N_{A}(x)$ of fixed donator/acceptor states at apposition $\mathrm{x}$ within the cell are assumed to be always completely ionized. Contrary, defects $N_{\text {trap }}(E)$ located at a specific energy $E$ within the bandgap of the semiconductor can be locally charged/uncharged within the system. Defects can be chosen to be either (1) acceptor-like Shockley-Read-Hall defects, (2) donor-like Shockley-Read-Hall defects or (3) dangling bond defects. Depending on the defect-type chosen, these defects can either be empty, singly occupied with electrons or even doubly occupied with electrons (in case of the dangling bond defect). Acceptor-like Shockley-Read-Hall defects are negatively charged, if occupied and neutral, if empty. Donor-like Shockley-Read-Hall defects are positively charged, if empty, and neutral, if occupied. Dangling bond defects are positively charged, if empty, neutral, if singly occupied and negatively charged, if doubly occupied.

Poisson's equation, which is to be solved within each layer, reads:

$$
\frac{\varepsilon_{0} \varepsilon_{r}}{q} \frac{\partial^{2} \varphi(x, t)}{\partial x^{2}}=p(x, t)-n(x, t)+N_{D}(x)-N_{A}(x)+\sum_{\text {trap }} \rho_{\text {trap }}(x, t)
$$

$q$ being the electron charge and $\varepsilon_{0}, \varepsilon_{r}$ being the absolute/relative dielectric constant. The defect density of charged defects $\rho_{\text {trap }}(x, t)$ will depend on the defect-type of the defect under consideration and on the local particle densities $n(x, t), p(x, t)$ within in the system. It is described by a trap density distribution function $N_{\text {trap }}(E)$ of the defect, specifying the amount of traps at an energy position $E$ within the bandgap and by some corresponding defect occupation functions $f_{0, \text { trap }}^{S R H}(E, x, t), f_{1, \text { trap }}^{S R H}(E, x, t), f_{+, \text {trap }}^{D B}(E, x, t), \quad f_{0, \text { trap }}^{D B}(E, x, t)$, $f_{-, \text {trap }}^{D B}(E, x, t)$, specifying the probability that traps with an energy position $E$ within the bandgap are empty or singly or doubly occupied with electrons. Thus $\rho_{\text {trap }}(x, t)$ equates to $\rho_{\text {trap }}(x, t)=-\int d E f_{1, \text { trap }}^{S R H}(E, x, t) N_{\text {trap }}(E)$ in case of acceptor-like Shockley-Read-Hall defects, $\rho_{\text {trap }}(x, t)=+\int d E f_{0, \text { trap }}^{\mathrm{SRH}}(E, x, t) N_{\text {trap }}(E)$ in case of donator-like Shockley-Read-Hall defects, $\rho_{\text {trap }}(x, t)=+\int d E\left(f_{+, \text {trap }}^{\mathrm{DB}}(E, x, t)-f_{-, \text {trap }}^{\mathrm{DB}}(E, x, t)\right) N_{\text {trap }}(E)$ in case of dangling bond defects. 
The explicit formulas for the defect occupation functions $f_{0, \text { trap }}^{S R H}(E, x, t), f_{1, \text { trap }}^{S R H}(E, x, t)$, $f_{+, \text {trap }}^{D B}(E, x, t), f_{0, \text { trap }}^{D B}(E, x, t), f_{-, \text {trap }}^{D B}(E, x, t)$ are described later within this text.

The one dimensional equations of continuity and transport for electrons and holes, which have to be solved within each layer, read:

$$
\begin{aligned}
& -\frac{1}{q} \frac{\partial j_{n}(x, t)}{\partial x}=G_{n}(x, t)-R_{n}(x, t)-\frac{\partial}{\partial t} n(x, t) \\
& +\frac{1}{q} \frac{\partial j_{p}(x, t)}{\partial x}=G_{p}(x, t)-R_{p}(x, t)-\frac{\partial}{\partial t} p(x, t)
\end{aligned}
$$

The electron/hole super-bandgap generation rates $G_{n}(x, t), G_{p}(x, t)$ have to be determined by optical modeling, the corresponding recombination rates $R_{n}(x, t), R_{p}(x, t)$ are described later in this text. The electron/hole currents $j_{n}(x, t), j_{p}(x, t)$ are driven by the gradient of the corresponding quasi Fermi energy $E_{F n}(x, t), E_{F p}(x, t)$. Using a Maxwell Boltzmann approximation for the Fermi-Dirac distribution function, the position dependent Fermi energies and the corresponding local electron/hole currents are explicitly:

$$
\begin{gathered}
E_{F n}(x, t)=E_{C}(x)+k T \ln \frac{n(x, t)}{N_{C}(x)}=-q \chi(x)+q \varphi(x, t)+k T \ln \frac{n(x, t)}{N_{C}(x)} \\
E_{F p}(x, t)=E_{V}(x)-k T \ln \frac{p(x, t)}{N_{V}(x)}=-q \chi(x)+q \varphi(x, t)-E_{g}(x)-k T \ln \frac{p(x, t)}{N_{V}(x)} \\
j_{n}(x, t)=q \mu_{n} n(x, t) \frac{\partial E_{F n}(x, t)}{\partial x} \\
j_{p}(x, t)=q \mu_{p} p(x, t) \frac{\partial E_{F p}(x, t)}{\partial x}
\end{gathered}
$$

with the corresponding electron/hole mobilities $\mu_{n}, \mu_{p}$, the electron affinity $\chi$, the bandgap $E_{g}$, the conduction/valence band energy $E_{C}, E_{V}$ and the effective conduction/valence band density of states $N_{C}, N_{V}$ of the semiconductor.

\section{Recombination}

Recombination from the conduction band into the valence band may occur directly, i.e. via radiative band to band recombination, $R_{n, p}^{B B}(x, t)$, or via Auger recombination, $R_{n, p}^{A}(x, t)$. It may also occur via defect states located within the bandgap of the semiconductor, i.e. via Shockley-Read-Hall recombination $R_{n, p}^{S H R}(x, t)$ or via dangling bond recombination, $R_{n, p}^{D B}(x, t)$ :

$$
R_{n, p}(x, t)=R_{n, p}^{B B}(x, t)+R_{n, p}^{A}(x, t)+R_{n, p}^{S H R}(x, t)+R_{n, p}^{D B}(x, t)
$$

\section{Optical sub-bandgap generation}

Optical sub-bandgap generation (for $h c / \lambda<E_{g}$ ) is calculated using Shockley-Read-Hall recombination statistics. A negative electron/hole SHR recombination rate $R_{n}^{S R H}(x, t)$, 
$R_{p}^{S R H}(x, t)$ means sub-bandgap generation of an electron/hole from a defect state (trap) into the conduction/valence band. Sub-bandgap generation can either be voltage driven and/or be driven by an optical excitation.

The SRH optical emission coefficients $e_{n, \text { optical }}^{\text {trap }}(E, x, t), e_{p, o p t i c a l}^{\text {trap }}(E, x, t)$ can be calculated from the optical electron/hole capture cross sections $\sigma_{n, o p t i c a l}^{\text {trap }}, \sigma_{p, o p t i c a l}^{\text {trap }}$ :

$$
\begin{aligned}
& e_{n, o p t i c a l}^{\text {trap }}(E, x, t)=\int_{\lambda_{\min }}^{\lambda_{\max }} d \lambda \sigma_{n, o p t i c a l}^{\text {trap }} N_{C} \Phi(\lambda, x, t) \vartheta\left(E_{C}-E-h c / \lambda\right) \\
& e_{p, \text { optical }}^{\text {trap }}(E, x, t)=\int_{\lambda_{\min }}^{\lambda_{\max }} d \lambda \sigma_{p, o p t i c a l}^{\text {trap }} N_{V} \Phi(\lambda, x, t) \vartheta\left(E-E_{V}-h c / \lambda\right)
\end{aligned}
$$

with $\Phi(\lambda, x, t)$ : spectral photon flux inside the semiconductor layers, of wavelength $\lambda$ at the position $x$ and at time $t, N_{C}, N_{V}$ : effective conduction/valence band density, $E_{C}, E_{V}$ : energy position of the conduction/valence band, and $\vartheta(E)$ : step function, $\vartheta(E)=1$ for $E \leq 0$, $\vartheta(E)=0$ for $E>0$.

Again, the minimum and maximum wavelengths $\lambda_{\min }, \lambda_{\max }$ for the integration are generally provided by the loaded spectral range of the incoming spectral photon flux, $\Phi_{0}(\lambda, t)$. However, if necessary, $\lambda_{\min }$ is modified in order to ensure that only sub-bandgap generation is considered: $\lambda_{\min } \geq h c / E_{g}$.

\section{Radiative recombination}

The radiative band to band rate constant $r^{B B}$ has to be specified in order to equate the radiative band to band recombination rates $R_{n, p}^{B B}(x, t)$. The resulting electron and hole recombination rates are always equal:

$$
R_{n, p}^{B B}(x, t)=r^{B B}\left\{n(x, t) p(x, t)-N_{C} N_{V} e^{-E_{g} / k T}\right\}
$$

In case of using the DC or AC calculation mode and neglecting second order terms in case of the AC calculation mode, this simplifies to

$$
\begin{gathered}
R_{n, p}^{B B}(x)=r^{B B}\left\{n^{D C}(x) p^{D C}(x)-N_{C} N_{V} e^{-E_{g} / k T}\right\} \\
R_{n, p}^{B B}(x, t)=R_{n, p}^{B B}(x)+\tilde{R}_{n, p}^{B B}(x) e^{i \omega t} \\
\tilde{R}_{n, p}^{B B}(x)=r^{B B} n^{D C}(x) \tilde{p}^{A C}(x)+r^{B B} p^{D C}(\mathrm{x}) \tilde{n}^{A C}(x)
\end{gathered}
$$

\section{Auger recombination}

The electron/hole Auger rate constants $r_{n}^{A}, r_{p}^{A}$ have to be specified in order to calculate the Auger recombination rates $R_{n, p}^{A}(x, t)$. Again, the resulting electron and hole recombination rates are always equal:

$$
R_{n, p}^{A}(x, t)=\left[r_{n}^{A} n(x, t)+r_{p}^{A} p(x, t)\right]\left\{n(x, t) p(x, t)-N_{C} N_{V} e^{-E_{g} / k T}\right\}
$$


In case of using the DC or AC calculation mode, neglecting second order terms within the AC calculation mode, this simplifies to

$$
\begin{gathered}
R_{n, p}^{A}(x)=\left[r_{n}^{A} n^{D C}(x)+r_{p}^{A} p^{D C}(x)\right]\left\{n^{D C}(x) p^{D C}(x)-N_{C} N_{V} e^{-E_{g} / k T}\right\} \\
R_{n, p}^{A}(x, t)=R_{n, p}^{A}(x)+\tilde{R}_{n, p}^{A}(x) e^{i \omega t} \\
\tilde{R}_{n, p}^{A}(x)=\left[r_{n}^{A} n^{D C}(x)^{2}+2 r_{p}^{A} n^{D C}(x) p^{D C}(x)\right] \tilde{p}^{A C}(x)+\left[r_{p}^{A} p^{D C}(x)^{2}+2 r_{n}^{A} n^{D C}(x) p^{D C}(x)\right] \tilde{n}^{A C}(x)
\end{gathered}
$$

\section{Shockley Read Hall recombination}

Shockley-Read-Hall recombination (Shockley \& Read, 1952) requires specifying the character (acceptor-like or donor-like), the capture cross sections $\sigma_{n}^{\text {trap }}, \sigma_{p}^{\text {trap }}, \sigma_{n, \text { optic }}^{\text {trap }}$, $\sigma_{p, o p t i c}^{\text {trap }}$ and the energetic distribution $N_{\text {trap }}(E)$ of the defect density within the bandgap of the semiconductor, of each defect. An arbitrary number of defects with either one of the following energetic distributions $N_{\text {trap }}(E)$ can be chosen:

1. point like distributed at a single energy $E_{\text {trap }}$ within the bandgap:

$$
N_{\text {trap }}(E)=N_{\text {trap }}^{\text {point }} \delta\left(E-E_{\text {trap }}\right)
$$

with $N_{\text {trap }}^{\text {point }}$ : defect density of the point like defect, $\delta(E)$ : delta function

2. constantly distributed within a specific region within the bandgap:

$$
N_{\text {trap }}(E)=\left(E_{\text {trap }}^{\text {end }}-E_{\text {trap }}^{\text {start }}\right) N_{\text {trap }}^{\text {const }} \vartheta\left(E-E_{\text {trap }}^{\text {end }}\right) \vartheta\left(E_{\text {trap }}^{\text {start }}-E\right)
$$

with $E_{\text {trap }}^{\text {start }}, E_{\text {trap }}^{\text {end }}$ : start and end energy of the energy interval within the bandgap, where a constant defect density is assumed, $N_{\text {trap }}^{\text {cont }}$ : constant defect density per energy, $\vartheta(E)$ : step function

3. exponentially decaying from the conduction/valence band into the bandgap:

$$
N_{\text {trap }}(E)=N_{\text {trap }}^{C \text {,tail }} e^{-\left(E_{C}-E\right) / E_{\text {trap }}^{\text {Ctail }}}, N_{\text {trap }}(E)=N_{\text {trap }}^{V, \text { tail }} e^{-\left(E-E_{V}\right) / E_{\text {trap }}^{\text {Vtail }}}
$$

i.e. conduction/valence band tail states, with $N_{\text {trap }}^{\text {C,tail }}, N_{\text {trap }}^{V \text {,tail }}$ : tail state density per energy at the conduction/valence band, $E_{\text {trap }}^{\text {C,tal }}, E_{\text {trap }}^{V \text {,tail }}$ : characteristic decay energy (Urbach energy) of the conduction/valence band tail state,

4. Gaussian distributed within the bandgap:

$$
N_{\text {trap }}(E)=\frac{N_{\text {trap }}^{d b}}{\sigma_{\text {trap }}^{d b} \sqrt{2 \pi}} e^{-\frac{\left(E-E_{\text {trap }}^{d b}\right)^{2}}{2 \sigma_{\text {trap }}^{2}}}
$$

i.e. dangling bond states, with $N_{\text {trap }}^{d b}$ : total dangling bond state density, $E_{\text {trap }}^{d b}$ : specific energy of the Gaussian dangling bond peak, $\sigma_{\text {trap }}^{d b}$ : standard deviation of the Gaussian dangling bond distribution. 
For each defect, electron/hole capture coefficients $c_{n, p}^{\text {trap }}$ are equated

$$
c_{n, p}^{\text {trap }}=v_{n, p} \sigma_{n, p}
$$

with $v_{n, p}$ : electron/hole thermal velocity, $\sigma_{n, p}$ : electron/hole capture cross section of the defect. The corresponding electron/hole emission coefficients $e_{n, p}^{\text {trap }}(E, x, t)$ are then given by:

$$
\begin{aligned}
& e_{n}^{\text {trap }}(E, x, t)=c_{n}^{\text {trap }} N_{C} e^{-\left(E_{C}-E\right) / k T}+e_{n, o p t i c}^{\text {trap }}(E, x, t) \\
& e_{p}^{\text {trap }}(E, x, t)=c_{p}^{\text {trap }} N_{V} e^{-\left(E-E_{V}\right) / k T}+e_{p, o p t i c}^{\text {trap }}(E, x, t)
\end{aligned}
$$

In case of using the DC or AC calculation mode, this simplifies to

$$
\begin{gathered}
\left.e_{n}^{\text {trap }}(E, x)=c_{n}^{\text {trap }} N_{C} e^{-\left(E_{C}-E\right) / k T}+\int d \lambda \sigma_{n, \text { trtic }}^{\text {trap }} N_{C} \Phi(\lambda, x) \vartheta\left(E_{C}-E-h c / \lambda\right) \quad \text { (DC mode }\right) \\
e_{p}^{\text {trap }}(E, x)=c_{p}^{\text {trap }} N_{V} e^{-\left(E-E_{V}\right) / k T}+\int d \lambda \sigma_{p, \text { optic }}^{\text {trap }} N_{V} \Phi(\lambda, x) \vartheta\left(E-E_{V}-h c / \lambda\right) \\
e_{n, p}^{\text {trap }}(E, x, t)=e_{n, p}^{\text {trap }}(E, x)+\tilde{e}_{n, p}^{\text {trap }}(E, x) e^{i \omega t} \quad(\text { AC mode }) \\
\tilde{e}_{n}^{\text {trap }}(E, x)=\int d \lambda \sigma_{n, o p t i c}^{\text {trap }} N_{C} \tilde{\Phi}(\lambda, x) \vartheta\left(E_{C}-E-h c / \lambda\right) \\
\tilde{e}_{p}^{\text {trap }}(E, x)=\int d \lambda \sigma_{p, \text { optic }}^{\text {trap }} N_{V} \tilde{\Phi}(\lambda, x) \vartheta\left(E-E_{V}-h c / \lambda\right)
\end{gathered}
$$

Finally, the Shockley-Read-Hall recombination rate due to the defects is

$$
\begin{aligned}
& R_{n}^{S R H}(x, t)=\sum_{\text {trap }} \int d E\left\{c_{n}^{\text {trap }} n(x, t) N_{\text {trap }}(E) f_{0, \text { trap }}^{S R H}(E, x, t)-e_{n}^{\text {trap }}(E, x, t) N_{\text {trap }}(E) f_{1, \text { trap }}^{S R H}(E, x, t)\right\} \\
& R_{p}^{S R H}(x, t)=\sum_{\text {trap }} \int d E\left\{c_{p}^{\text {trap }} p(x, t) N_{\text {trap }}(E) f_{1, \text { trap }}^{S R H}(E, x, t)-e_{p}^{\text {trap }}(E, x, t) N_{\text {trap }}(E) f_{0, \text { trap }}^{S R H}(E, x, t)\right\}
\end{aligned}
$$

In case of using the DC or AC calculation mode, neglecting second order terms and assuming zero optical emission coefficients within the AC calculation mode (actual stage of the AFORS-HET development at the moment) this simplifies to

$$
\begin{gathered}
R_{n}^{S R H}(x)=\sum_{\text {trap }} \int d E\left\{c_{n}^{\text {trap }} n^{D C}(x) N_{\text {trap }}(E) f_{0, \text { trap }}^{S R H}(E, x)-e_{n}^{\text {trap }}(E, x) N_{\text {trap }}(E) f_{1, \text { trap }}^{S R H}(E, x)\right\} \\
R_{p}^{S R H}(x)=\sum_{\text {trap }} \int d E\left\{c_{p}^{\text {trap }} p^{D C}(x) N_{\text {trap }}(E) f_{1, \text { trap }}^{S R H}(E, x)-e_{p}^{\text {trap }}(E, x) N_{\text {trap }}(E) f_{0, \text { trap }}^{S R H}(E, x)\right\} \\
R_{n, p}^{S R H}(x, t)=R_{n, p}^{S R H}(x)+\tilde{R}_{n, p}^{S R H}(x) e^{\text {iøt }} \\
\tilde{R}_{n}^{S R H}(x)=\sum_{\text {trap }} \int d E\left\{c_{n}^{\text {trap }} N_{\text {trap }}(E) f_{0, \text { trap }}^{S R H}(E, x) \tilde{n}^{A C}(x)-\left(c_{n}^{\text {trap }}+e_{n}^{\text {trap }}(E, x)\right) N_{\text {trap }}(E) \tilde{f}_{1, \text { trap }}^{S R H}(E, x)\right\} \\
\tilde{R}_{p}^{S R H}(x)=\sum_{\text {trap }} \int d E\left\{c_{p}^{\text {trap }} N_{\text {trap }}(E) f_{1, \text { trap }}^{S R H}(E, x) \tilde{p}^{A C}(x)+\left(c_{p}^{\text {trap }}+e_{p}^{\text {trap }}(E, x)\right) N_{\text {trap }}(E) \tilde{f}_{1, \text { trap }}^{\text {SRH }}(E, x)\right\}
\end{gathered}
$$


A positive electron/hole SHR recombination rate means recombination of an electron/hole from the conduction/valence band into the defect state (trap), a negative electron/hole SHR recombination rate means sub-bandgap generation of an electron/hole from the defect state (trap) into the conduction/valence band.

\section{Dangling bond recombination}

To calculate charge state and recombination of dangling bond defects in amorphous silicon the most exact description developed by Sah and Shockley (Sah \& Shockley, 1958) is used. Three different occupation functions $f_{+, \text {trap }}^{D B}(E, x, t), f_{0, \text { trap }}^{D B}(E, x, t), f_{-, \text {trap }}^{D B}(E, x, t)$ for the positively, neutral and negatively charge states have to be derived, corresponding to the empty, single or double occupied electronic state. Four capture/emission processes with the capture cross sections $\sigma_{n}^{+}, \sigma_{p}^{0}, \sigma_{n}^{0}, \sigma_{p}^{-}$have to be defined as can be seen in Fig. 3 . The two transition energies $E_{0 /-}, E_{+/ 0}$ are separated by the correlation energy $\mathrm{U}$, which accounts for the fact that the capture-emission process is influenced by the charge state of the dangling or by rearrangement of the lattice in the surrounding.

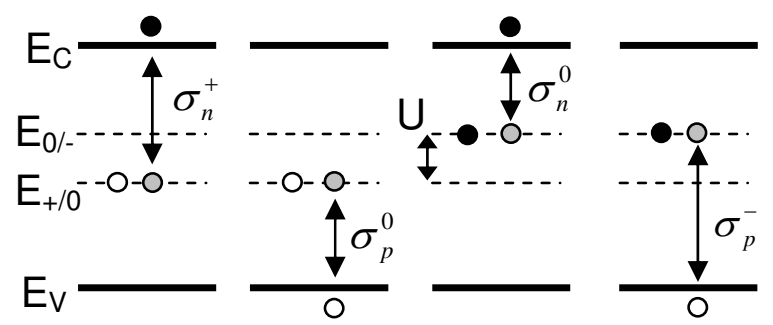

Fig. 3. dangling bond recombination according to Sah and Shockley

For each defect, electron/hole capture coefficients $c_{n / p,+/ 0 /-}^{\text {trap }}$ are calculated with the specified electron/hole thermal velocity $v_{n / p}$ :

$$
c_{n / p,+/ 0 /-}^{\text {trap }}=v_{n / p} \sigma_{n / p}^{+/ 0 /-}
$$

The emission coefficients for the DC calculation mode, neglecting optical emission are given by:

$$
\begin{gathered}
e_{n, 0}^{\text {trap }}(E, x)=\frac{1}{2} c_{n,+}^{\text {trap }} N_{C} e^{-\left(E_{C}-E\right) / k T} \\
e_{p,+}^{\text {trap }}(E, x)=2 c_{p, 0}^{\text {trap }} N_{V} e^{-\left(E-E_{V}\right) / k T} \\
e_{n,-}^{\text {trap }}(E, x)=2 c_{n, 0}^{\text {trap }} N_{C} e^{-\left(E_{C}-(E+U)\right) / k T} \\
e_{p, 0}^{\text {trap }}(E, x)=\frac{1}{2} c_{p,-}^{\text {trap }} N_{V} e^{-\left(E+U-E_{V}\right) / k T}
\end{gathered}
$$

Finally the dangling bond recombination coefficients are given by:

$$
\begin{array}{r}
R_{n}^{D B}(x)=\sum_{\text {trap }} \int d E\left\{c_{n,+}^{\text {trap }} n^{D C}(x) N_{\text {trap }}(E) f_{+, \text {trap }}^{D B}(E, x)-e_{n, 0}^{\text {trap }}(E, x) N_{\text {trap }}(E) f_{0, \text { trap }}^{D B}(E, x)+\right. \\
\left.c_{n, 0}^{\text {trap }} n^{D C}(x) N_{\text {trap }}(E) f_{0, \text { trap }}^{D B}(E, x)-e_{n,-}^{\text {trap }}(E, x) N_{\text {trap }}(E) f_{-, \text {trap }}^{D B}(E, x)\right\}
\end{array}
$$




$$
\begin{array}{r}
R_{p}^{D B}(x)=\sum_{\text {trap }} \int d E\left\{c_{p,-}^{\text {trap }} p^{D C}(x) N_{\text {trap }}(E) f_{-, \text {trap }}^{D B}(E, x)-e_{p, 0}^{\text {trap }}(E, x) N_{\text {trap }}(E) f_{0, \text { trap }}^{D B}(E, x)+\right. \\
\left.c_{p, 0}^{\text {trap }} p^{D C}(x) N_{\text {trap }}(E) f_{0, \text { trap }}^{D B}(E, x)-e_{p,+}^{\text {trap }}(E, x) N_{\text {trap }}(E) f_{+, \text {trap }}^{D B}(E, x)\right\}
\end{array}
$$

Dangling bond recombination is still under development. Especially time dependent recombination and optical defect to band emissions are not implemented at the current state of AFORS-HET development.

\section{Defect occupation functions}

The defect occupation functions $f_{0, \text { trap }}^{S R H}(E, x, t), f_{1, \text { trap }}^{S R H}(E, x, t), f_{+, \text {trap }}^{D B}(E, x, t), f_{0, \text { trap }}^{D B}(E, x, t)$, $f_{-, \text {trap }}^{D B}(E, x, t)$ specify the probability for a specific defect (either Shockley-Read-Hall or dangling bond) that traps with an energy position $E$ within the bandgap of the semiconductor are empty or singly or even doubly occupied with electrons.

In case of using the DC or AC calculation mode, they can be explicitly expressed in terms of the local particle densities $n^{D C}(x), p^{D C}(x), \tilde{n}^{A C}(x), \tilde{p}^{A C}(x)$. In case of using the TR calculation mode, the defect occupation functions are generally determined by additional differential equations. Transient DB defect occupation functions have not been implemented in AFORS-HET yet (actual stage of AFORS-HET development).

\section{Shockley Read Hall defect occupation functions}

A Shockley-Read-Hall defect can be either empty or occupied by an electron, thus

$$
f_{0, \text { trap }}^{S R H}(E, x, t)+f_{1, \text { trap }}^{S R H}(E, x, t)=1
$$

The Shockley-Read-Hall defect occupation function $f_{1, \text { trap }}^{S R H}(E, x, t)$ for electrons will be explicitly stated in case of using the EQ, DC, AC or the TR calculation mode. The ShockleyRead-Hall defect occupation function $f_{0, \text { trap }}^{S R H}(E, x, t)$ can then directly be equated.

Generally, a local change of the trapped charge stored in SRH defects must be determined by the difference between the local electron and hole SRH recombination rates:

$$
\frac{d}{d t} \rho_{\text {trap }}(x, t)=R_{p}^{S H R}(x, t)-R_{n}^{S H R}(x, t)
$$

This defines for each defect an additional differential equation for its SHR defect occupation function $f_{1, \text { trap }}^{S R H}(E, x, t)$ with respect to its time derivative:

$$
\frac{d}{d t} f_{1, \text { trap }}^{\text {SRH }}(E, x, t)=\left(c_{n}^{\text {trap }} n(x, t)+e_{p}^{\text {trap }}(E, x, t)\right)\left(1-f_{1, \text { trap }}^{\text {SRH }}(E, x, t)\right)-\left(c_{p}^{\text {trap }} p(x, t)+e_{n}^{\text {trap }}(E, x, t)\right) f_{1, \text { trap }}^{\text {SRH }}(E, x, t)
$$

In case of using the EQ or the DC calculation mode, the time derivative vanishes, and an explicit expression for the SHR defect occupation function, $f_{1, \text { trap }}^{S R H}(E, x)$, which is no longer time dependant, can be derived:

$$
f_{1, \text { trap }}^{S R R}, D C(E, x)=\frac{c_{n}^{\text {trap }} n^{D C}(x)+e_{p}^{\text {trap }}(E, x)}{c_{n}^{\text {trap }} n^{D C}(x)+e_{n}^{\text {trap }}(E, x)+c_{p}^{\text {trap }} p^{D C}(x)+e_{p}^{\text {trap }}(E, x)}
$$


Of course, in case of using the EQ calculation mode, the SHR defect occupation function could be also equivalently be described by the Fermi-Dirac distribution function, $f_{1, \text { trap }}^{S R H, D C}(E, x)=f_{1, \text { trap }}^{S R H, E Q}(E, x)$, which implicitly determines the position independent Fermi energy $E_{F}$.

$$
f_{1, \text { trap }}^{S R H, E Q}(E, x)=\frac{1}{1+e^{\frac{E-E_{F}}{k T}}}
$$

In case of using the AC calculation mode, the differential equation (\#) can be explicitly solved, assuming time independent optical emission coefficients within the AC calculation mode (actual stage of the AFORS-HET development at the moment) and assuming the time dependencies $n(x, t)=n^{D C}(x)+\tilde{n}^{A C}(x) e^{i \omega t}, \quad p(x, t)=p^{D C}(x)+\tilde{p}^{A C}(x) e^{i \omega t}$. Neglecting second order terms, one gets for the SHR defect occupation function in the AC calculation mode, $f_{1, \text { trap }}^{S R H, A C}(E, x, t)$ :

$$
\begin{gathered}
f_{1, \text { trap }}^{S R H C}(E, x, t)=f_{1, \text { trap }}^{S R H, D C}(E, x)+\tilde{f}_{1, \text { trap }}^{S R H, A C}(E, x) e^{i \omega t} \\
\tilde{f}_{1, \text { trap }}^{S R H, A C}(E, x)=\frac{c_{n}^{\text {trap }} f_{0, \text { trap }}^{S R H, D C}(E, x) \tilde{n}^{A C}(x)-c_{p}^{\text {trap }} f_{1, \text { trap }}^{S R H}(E, x) \tilde{p}^{A C}(x)}{c_{n}^{\text {trap }} n^{D C}(x)+e_{n}^{\text {trap }}(E, x)+c_{p}^{\text {trap }} p^{D C}(x)+e_{p}^{\text {trap }}(E, x)+i \omega}
\end{gathered}
$$

In case of using the TR calculation mode, the transient SRH defect occupation function $f_{1, \text { trap }}^{S R H}\left(E, x, t_{i+1}\right)$ at the time step $t_{i+1}$ for an evolution of the system from the time point $t_{i}$ towards the time point $t_{i+1}$ can be stated by solving the differential equation (\#) using a full implicit time discretisation scheme with respect to the particle densities and the emission rates:

$$
\begin{aligned}
& \frac{d}{d t} f_{1, \text { trap }}^{\text {SRH } T R}(E, x, t) \\
& =\left(c_{n}^{\text {trap }} n\left(x, t_{i+1}\right)+e_{p}^{\text {trap }}\left(E, x, t_{i+1}\right)\right)\left(1-f_{1, \text { trap }}^{\text {SRH,TR }}(E, x, t)\right)-\left(c_{p}^{\text {trap }} p\left(x, t_{i+1}\right)+e_{n}^{\text {trap }}\left(E, x, t_{i+1}\right)\right) f_{1, \text { trap }}^{\text {SR }, \text { TR }}(E, x, t)
\end{aligned}
$$

An analytical solution of this differential equation leads to:

$$
f_{1, \text { trap }}^{S R H, T R}\left(E, x, t_{i+1}\right)=f_{1, \text { trap }}^{S R H, D C t r}\left(E, x, t_{i+1}\right)-\frac{f_{1, t \text { trap }}^{S R H, D C t r}\left(E, x, t_{i+1}\right)-f_{1, \text { trap }}^{S R H, T R}\left(E, x, t_{i}\right)}{\left.e^{d t\left(c_{n}^{\text {trap }} n\left(x, t_{i+1}\right)+e_{n}^{\text {trap }}\left(E, x, t_{i+1}\right)+c_{p}^{\text {trap }} p\left(x, t_{i+1}\right)+e_{p}\left(E, x, t_{i+1}\right)\right.}\right)}
$$

with

$$
f_{S R H}^{D C t r}\left(E, x, t_{i+1}\right)=\frac{c_{n}^{\text {trap }} n\left(x, t_{i+1}\right)+e_{p}^{\text {trap }}\left(E, x, t_{i+1}\right)}{c_{n}^{\text {trap }} n\left(x, t_{i+1}\right)+e_{n}^{\text {trap }}\left(E, x, t_{i+1}\right)+c_{p}^{\text {trap }} p\left(x, t_{i+1}\right)+e_{p}^{\text {trap }}\left(E, x, t_{i+1}\right)}
$$

In the steady-state limit, i.e. for Limes $d t \rightarrow \infty, d t=t_{i+1}-t_{i}$, this formula converts to the well known steady state SRH defect occupation function $f_{1, \text { trap }}^{S R H, D C}(E, x)=f_{1, \text { trap }}^{S R H, D C t r}\left(E, x, t_{i+1}\right)$.

\section{Dangling bond defect occupation functions}

A dangling bond defect can be either empty or singly or doubly occupied by an electron, hereby being in its positive, neutral or negative charged state, thus 


$$
f_{+, \text {trap }}^{D B}(E, x, t)+f_{0, \text { trap }}^{D B}(E, x, t)+f_{-, \text {trap }}^{D B}(E, x, t)=1
$$

For the EQ and DC calculation mode the occupation functions are given by:

$$
\begin{aligned}
& f_{+}^{D B}(E, x)=\frac{P^{0} P^{-}}{N^{+} P^{-}+P^{0} P^{-}+N^{+} N^{0}} \\
& f_{0}^{D B}(E, x)=\frac{P^{-} N^{+}}{N^{+} P^{-}+P^{0} P^{-}+N^{+} N^{0}} \\
& f_{-}^{D B}(E, x)=\frac{N^{0} N^{+}}{N^{+} P^{-}+P^{0} P^{-}+N^{+} N^{0}}
\end{aligned}
$$

where:

$$
\begin{aligned}
& N^{+}=c_{n}^{+} n^{D C}+e_{p}^{+} \\
& P^{0}=c_{p}^{0} p^{D C}+e_{n}^{0} \\
& N^{0}=c_{n}^{0} n^{D C}+e_{p}^{0} \\
& P^{-}=c_{p}^{-} p^{D C}+e_{n}^{-}
\end{aligned}
$$

Dangling bond defects are still under development and especially time dependent dangling bond occupation functions (to be used in the AC or TR calculation mode) are not implemented at the current state of AFORS-HET development.

\subsubsection{Bulk model: crystalline silicon}

If the bulk model "crystalline silicon" is used for a semiconductor layer, most layer input parameter as described in chapter 3.2 are calculated from the doping densities and the defect densities of crystalline silicon.

Thus a doping and temperature dependent material parameterization for crystalline silicon can be undertaken, i.e. it is possible to specify (1) the temperature dependence of the intrinsic carrier concentration of crystalline silicon (intrinsic carrier density model), (2) the doping dependence of the electron/hole mobilities in crystalline silicon (mobility model), (3) the doping dependence of the Auger recombination in crystalline silicon or even its dependence on local excess carriers (Auger recombination models), (4) the doping and the temperature dependence of the Shockley-Read-Hall recombination in crystalline silicon (SRH lifetime model) and (5) doping dependence of the bandgap in crystalline silicon (bandgap narrowing model). All these models are similar to the numerical computer simulation program for crystalline silicon solar cells PC1D (Basore \& Clugston, 1997).

Only one single neutral SRH defect at a certain energy position E within the bandgap is assumed. The doping densities $N_{D}, N_{A}$ and the amount of traps $N_{\text {trap }}$ are specified by the user, all other layer input parameters are calculated according to the above mentioned models. 


\subsection{Electrical calculation - interfaces: semiconductor/semiconductor interface models}

Each interface between two adjacent semiconductor layers can be described by three different interface models: (1) interface model: "no interface", (2) interface model: "driftdiffusion interface" and (3) interface model: "thermionic emission interface". If "no interface" is chosen, the transport across the interface is treated in complete analogy to the "drift diffusion" interface model, however, no interface defects can be specified. The "drift diffusion" interface model models the transport across the heterojunction interface in the same way as in the bulk layers, thereby assuming a certain interface thickness. The "thermionic emission" interface model treats a real interface which interacts with both adjacent semiconductor layers.

\subsubsection{Interface model: no interface}

Per default, the electron and hole currents across the semiconductor/semiconductor interface are assumed to be driven by drift diffusion, with no interface defects present at the interface.

The drift diffusion model assumes an interface layer of a certain thickness (which is given by the specified grid point to boundary distance within the numerical settings of AFORS-HET). Within this interface layer, the material properties change linearly from semiconductor I to semiconductor II. The elelctron/hole currents across the heterojunction interface can then be treated like in the bulk of a semiconductor layer (drift diffusion driven).

Denoting $x_{i t}^{I}, x_{i t}^{I I}$ the positions directly adjacent to the semiconductor I/II located left/right to the interface, $\mu_{n, p}^{I}, \mu_{n, p}^{I I}$ the mobilities of the two adjacent semiconductors, and $\mu_{n, p}^{i t}=\frac{\mu_{n, p}^{I}+\mu_{n, p}^{I I}}{2}, n^{i t}=\frac{n\left(x_{i t}^{I}\right)+n\left(x_{i t}^{I I}\right)}{2}, p^{i t}=\frac{p\left(x_{i t}^{I}\right)+p\left(x_{i t}^{I I}\right)}{2}$, the corresponding electron $/$ hole currents across the interface are (like in the bulk):

$$
\begin{aligned}
& j_{n}^{i t}(t)=q \mu_{n}^{i t} n^{i t}(t) \frac{\partial E_{F n}(x, t)}{\partial x} \\
& j_{p}^{i t}(t)=q \mu_{p}^{i t} p^{i t}(t) \frac{\partial E_{F p}(x, t)}{\partial x}
\end{aligned}
$$

\subsubsection{Interface model: drift-diffusion interface}

The electron and hole currents across the semiconductor/semiconductor interface are assumed to be driven by drift diffusion, with additional interface defects present at the interface. Half of the interface states can be occupied by electrons or holes from semiconductor I, the other half from semiconductor II.

The interface defects (given in $\mathrm{cm}^{-2}$ ) are distributed homogeneously within the interface layer (per $\mathrm{cm}^{-3}$ ). I.e. the specified interface defect density $N_{i t}(E)$ is converted into a homogeneous layer defect density of the interface layer, $N_{t}(E)$, selectively according to one of the two following formulas, either $N_{t}(E)=\frac{N_{i t}(E)}{d_{i t}}$ or $N_{t}(E)=\left(N_{i t}(E)\right)^{\frac{3}{2}}$. Thus half of the defects specified are recombination active within semiconductor I, the other half within 
semiconductor II. The calculation of the electron/hole currents across the heterojunction interface is then performed in the same way as when using the interface model "no interface" described above.

\subsubsection{Interface model: thermionic emission interface}

The electron and hole currents across the interface are assumed to be driven by thermionic emission (Anderson, 1962; Sze \& Kwok, 2007). Additional interface defects can be present at the interface. These states can be filled with electrons or holes from both sides of the interface.

Lets denote $x_{i t}^{I}, x_{i t}^{I I}$ the positions directly adjacent to the semiconductor I/II located left/right to the interface, $\chi^{I, I I}, E_{g}^{I, I I}, v_{n, p}^{I, I I}$ the electron affinity, the bandgap and the thermal velocities of the two semiconductors. According to Anderson theory (Anderson, 1962) the conduction/valence band offsets $\Delta E_{C}, \Delta E_{V}$, which determine the energetic barrier of the heterojunction interface to be overcome by thermionic emission, are:

$$
\begin{gathered}
\Delta E_{C}=\chi^{I I}-\chi^{I} \\
\Delta E_{V}=E_{g}^{I I}-E_{g}^{I}+\chi^{I I}-\chi^{I}
\end{gathered}
$$

The sign convention is such, that $\Delta E_{C}, \Delta E_{V}$ is negative if $E_{C}, E_{V}$ drops from the left side to the right side of the interface. The thermionic emission currents across the heterojunction interface $j_{n}^{T E, I \rightarrow I I}(t), j_{p}^{T E, I \rightarrow I I}(t), j_{n}^{T E, I I \rightarrow I}(t), j_{p}^{T E, I I \rightarrow I}(t)$ are then explicitly written using the $\vartheta(E)$ step function, $\vartheta(E)=1$ for $E \leq 0, \vartheta(E)=0$ for $E>0$ :

$$
\begin{array}{ll}
j_{n}^{T E, I \rightarrow I I}(t)=v_{n}^{I} n\left(x_{i t}^{I}, t\right) e^{-\frac{\left|\Delta E_{C}\right|}{k T} \vartheta\left(-\Delta E_{C}\right)}, & j_{n}^{T E, I I \rightarrow I}(t)=v_{n}^{I I} n\left(x_{i t}^{I I}, t\right) e^{-\frac{\left|\Delta E_{C}\right|}{k T} \vartheta\left(\Delta E_{C}\right)} \\
j_{p}^{T E, I \rightarrow I I}(t)=v_{p}^{I} p\left(x_{i t}^{I}, t\right) e^{-\frac{\left|\Delta E_{V}\right|}{k T} \vartheta\left(\Delta E_{V}\right)}, & j_{p}^{T E, I I \rightarrow I}(t)=v_{p}^{I I} p\left(x_{i t}^{I I}, t\right) e^{-\frac{\left|\Delta E_{V}\right|}{k T} \vartheta\left(-\Delta E_{V}\right)}
\end{array}
$$

The netto electron/hole current across the interface due to thermionic emission $j_{n}^{i t}, j_{p}^{i t}$, is:

$$
j_{n}^{i t}=j_{n}^{T E, I \rightarrow I I}-j_{n}^{T E, I I \rightarrow I}, \quad j_{p}^{i t}=j_{p}^{T E, I \rightarrow I I}-j_{p}^{T E, I I \rightarrow I}
$$

Additional to the thermionic emission process across the heterojunction interface, there is recombination due to the interface defects. The interface electron/hole recombination rates from both sides of the interface $R_{n, I}^{i t}(t), R_{n, I I}^{i t}(t), R_{p, I}^{i t}(t), R_{p, I I}^{i t}(t)$, are described with SRH recombination:

$$
\begin{aligned}
R_{n, I}^{i t}(t) & =\sum_{\text {trap }} \int d E\left\{c_{n, I}^{i t} n\left(x_{i t}^{I}, t\right) N_{\text {trap }}^{i t}(E)\left(1-f_{\text {trap }}^{i t}(E, t)\right)-e_{n, I}^{i t}(E, t) N_{\text {trap }}^{i t}(E) f_{\text {trap }}^{i t}(E, t)\right\} \\
R_{n, I I}^{i t}(t) & =\sum_{\text {trap }} \int d E\left\{c_{n, I I}^{i t} n\left(x_{i t}^{I I}, t\right) N_{\text {trap }}^{i t}(E)\left(1-f_{\text {trap }}^{i t}(E, t)\right)-e_{n, I I}^{i t}(E, t) N_{\text {trap }}^{i t}(E) f_{\text {trap }}^{i t}(E, t)\right\} \\
R_{p, I}^{i t}(t) & =\sum_{\text {trap }} \int d E\left\{c_{p, I}^{i t} p\left(x_{i t}^{I}, t\right) N_{\text {trap }}^{i t}(E) f_{\text {trap }}^{i t}(E, t)-e_{p, I}^{i t}(E, t) N_{\text {trap }}^{i t}(E)\left(1-f_{\text {trap }}^{i t}(E, x, t)\right)\right\}
\end{aligned}
$$




$$
R_{p, I I}^{i t}(t)=\sum_{\text {trap }} \int d E\left\{c_{p, I I}^{i t} p\left(x_{i t}^{I I}, t\right) N_{\text {trap }}^{i t}(E) f_{\text {trap }}^{i t}(E, t)-e_{p, I I}^{i t}(E, t) N_{\text {trap }}^{i t}(E)\left(1-f_{\text {trap }}^{i t}(E, x, t)\right)\right\}
$$

Thus, recombination at the interface is treated equivalently to bulk SRH recombination, with two exceptions: The interface defect density $N_{\text {trap }}^{i t}(E)$ is now given in defects per $\mathrm{cm}^{-2} \mathrm{eV}^{-1}$ instead of $\mathrm{cm}^{-3} \mathrm{eV}^{-1}$, consequently, interface recombination is now a recombination current, given in $\mathrm{cm}^{-2} \mathrm{~s}^{-1}$, instead of $\mathrm{cm}^{-3} \mathrm{~s}^{-1}$. Furthermore, the interface defect distribution function $f_{\text {trap }}^{i t}(E, t)$ changes compared to the bulk defect distribution function $f_{\text {trap }}(E, x, t)$, as the interface states can interact with both adjacent semiconductors. For the EQ or DC calculation mode, one gets explicitly :

$$
\begin{aligned}
& f_{\text {trap }}^{i t, D C}(E)=f_{\text {trap }}^{i t, E Q}(E)= \\
& \frac{c_{n, I}^{i t} n\left(x_{i t}^{I}\right)+c_{n, I I}^{i t} n\left(x_{i t}^{I I}\right)+e_{p, I}^{i t}(E)+e_{p, I I}^{i t}(E)}{c_{n, I}^{i t} n\left(x_{i t}^{I}\right)+c_{n, I I}^{i t} n\left(x_{i t}^{I I}\right)+c_{p, I}^{i t} p\left(x_{i t}^{I}\right)+c_{p, I I}^{i t} p\left(x_{i t}^{I I}\right)+e_{n, I}^{i t}(E)+e_{n, I I}^{i t}(E)+e_{p, I}^{i t}(E)+e_{p, I I}^{i t}(E)}
\end{aligned}
$$

Using the AC calculation mode, one gets:

$$
f_{\text {trap }}^{i t, A C}(E, t)=f_{\text {trap }}^{i t, D C}(E)+\tilde{f}_{\text {trap }}^{i t, A C}(E) e^{i \omega t}
$$

$$
\begin{aligned}
& \tilde{f}_{\text {trap }}^{i t, A C}(E)= \\
& \frac{\left\{1-f_{\text {trap }}^{i t, D C}(E)\right\}\left\{c_{n, I}^{i t} \tilde{n}^{A C}\left(x_{i t}^{I}\right)+c_{n, I I}^{i t} \tilde{n}^{A C}\left(x_{i t}^{I I}\right)\right\}-f_{\text {trap }}^{i t, D C}(E)\left\{c_{p, I}^{i t} \tilde{p}^{A C}\left(x_{i t}^{I}\right)+c_{p, I I}^{i t} \tilde{p}^{A C}\left(x_{i t}^{I I}\right)\right\}}{c_{n, I}^{i t} n\left(x_{i t}^{I}\right)+c_{n, I I}^{i t} n\left(x_{i t}^{I I}\right)+c_{p, I}^{i t} p\left(x_{i t}^{I}\right)+c_{p, I I}^{i t} p\left(x_{i t}^{I I}\right)+e_{n, I}^{i t}(E)+e_{n, I I}^{i t}(E)+e_{p, I}^{i t}(E)+e_{p, I I}^{i t}(E)+i \omega}
\end{aligned}
$$

Within the actual stage of AFORS-HET development, interface states described by thermionic emission are only implemented within the EQ, DC and AC calculation mode, i.e. the transient defect distribution function of such states has not been implemented yet.

The heterojunction interface itself is treated as a boundary condition for the differential equations describing the semiconductor layers. Thus, six boundary conditions for the potential and the electron/hole currents at each side of the interface have to be stated, i.e.:

The potential is assumed to be equal on both sides of the interface (thereby neglecting interface dipoles):

1. $\varphi\left(x_{i t}^{I}\right)=\varphi\left(x_{i t}^{I I}\right)$

The total charge stored in the interface states is equal to the difference in the dielectric displacements (a consequence of the Gauss law applied to the Poisson equation)

2. $\left.\varepsilon_{0} \varepsilon_{r}^{I} \frac{\partial \varphi(x)}{\partial x}\right|_{x_{i t}^{I}}-\left.\varepsilon_{0} \varepsilon_{r}^{I I} \frac{\partial \varphi(x)}{\partial x}\right|_{x_{i t}^{I I}}=q \sum_{\text {defects }} \rho_{i t}$

The total current across the heterojunction interface $j_{\text {ges }}^{i t}(t)=j_{n}^{i t}(t)+j_{p}^{i t}(t)$ under steady-state conditions is equal to the constant (that is position independent) total current left (or right) to the interface

3. $j_{n}^{i t}(t)+j_{p}^{i t}(t)=j_{n}\left(x_{i t}^{I}, t\right)+j_{p}\left(x_{i t}^{I}, t\right)$

The total electron/hole recombination rate from both sides of the interface is equal for electrons and holes (valid only for EQ, DC and AC calculation mode) 
4. $R_{n, I}^{i t}(t)+R_{n, I I}^{i t}(t)=R_{p, I}^{i t}(t)+R_{p, I I}^{i t}(t)$

The electron/hole current left to the interface is equal to the netto electron/hole current across the heterojunction interface plus the interface recombination current

5. $j_{n}\left(x_{i t}^{I}, t\right)=j_{n}^{i t}(t)+R_{n, I}^{i t}(t)$

6. $j_{p}\left(x_{i t}^{I}, t\right)=j_{p}^{i t}(t)-R_{p, I}^{i t}(t)$

\subsection{Electrical calculation - boundaries: front/back contact to semiconductor models}

The electrical front/back contacts of the semiconductor stack are usually assumed to be metallic, in order to be able to withdraw a current. However, they may also be insulating in order to be able to simulate some specific measurement methods like for example quasi steady state photoconductance (QSSPC) or surface photovoltage (SPV). So far, four different boundary models for the interface between the contact and the semiconductor adjacent to the contact can be chosen: (1) "flatband metal/semiconductor contact" (2) "Schottky metal/semiconductor contact", (3) "insulator/semiconductor contact", (4) "metal/insulator/semiconductor contact". The boundaries serve as a boundary condition for the system of differential equations describing the semiconductor stack, thus three boundary conditions for the potential and the electron/hole currents at the front and at the back side of the stack have to be stated.

\subsubsection{Boundary model: flatband metal/semiconductor contact}

Per default, an idealized flatband metal/semiconductor contact is assumed at the boundaries. That is, only the effective electron/hole surface recombination velocities $S_{n / p}^{\text {front } / \text { back }}$ have to be specified. The metal work function of the front/back contact, $\phi^{\text {front/back }}$ is calculated in a way, that flatband conditions are reached according to Schottky theory (Sze \& Kwok, 2007). Normally, flatband conditions are calculated within the thermal equilibrium EQ calculation mode, however, in case of using the DC, AC or TR calculation mode with an external illumination (optical super bandgap generation) enabled, they are recalculated in order to ensure flatband conditions independent from the applied illumination.

The interface between the metallic front/back contact and the semiconductor is treated as a boundary condition for the differential equations describing the semiconductor layers. Thus, for each contact, three boundary conditions involving the potential and electron/hole densities adjacent to the contact have to be stated. Denoting $x_{i t}^{\text {front }}, x_{i t}^{\text {back }}$ the position within the semiconductor directly adjacent to the metallic contact, these are:

The electric potential is fixed to zero at one contact (for example the back contact).

1.a $\varphi\left(x_{i t}^{\text {back }}, t\right)=0$

At the other contact (for example the front contact) the external applied cell voltage $V_{\text {ext }}(t)$ or the external applied current density $j_{e x t}(t)$ through the cell is specified (voltage controlled or current controlled calculation). The external solar cell resistances, i.e. the series resistance $R_{e x t}^{S}$ and the parallel resistance $R_{\text {ext }}^{P}$, which can optionally be specified, will affect the internal cell voltage $V_{\text {int }}(t)$ at the boundary of the semiconductor stack and also the position independent internal current density $j_{\text {int }}(t)$ through the semiconductor stack. 
In case of a voltage controlled calculation, the internal cell voltage can be expressed by the specified external cell voltage $V_{\text {ext }}(t)$ and the position independent internal cell current $j_{\text {int }}(t)=$ Const $(t)=j_{n}(x, t)+j_{p}(x, t)$ :

$$
V_{\text {int }}(t)=V_{e x t}(t)+\frac{j_{\text {int }}(t) R_{e x t}^{S}}{1+\frac{R_{e x t}^{S}}{R_{e x t}^{P}}}=V_{\text {ext }}(t)+\frac{\left\{j_{n}\left(x_{i t}^{\text {front }}, t\right)-j_{p}\left(x_{i t}^{\text {front }}, t\right)\right\} R_{e x t}^{S}}{1+\frac{R_{e x t}^{S}}{R_{e x t}^{P}}}
$$

Thus the potential at other contact can be specified:

1.b $\varphi\left(x_{i t}^{\text {front }}, t\right)=\phi^{\text {front }}-\phi^{\text {back }}+V_{\text {int }}(t)$

In case of a current controlled calculation, the internal cell current density can be expressed by the specified external cell current density $j_{e x t}(t)$ and the internal cell voltage $V_{\text {int }}(t)=\varphi\left(x_{i t}^{\text {front }}, t\right)-\varphi\left(x_{i t}^{\text {back }}, t\right)-\phi^{\text {front }}+\phi^{\text {back }}$ :

$$
j_{\text {int }}(t)=j_{\text {ext }}(t)+\frac{V_{\text {int }}(t)}{R_{e x t}^{P}}=j_{\text {ext }}(t)+\frac{\varphi\left(x_{i t}^{\text {front }}, t\right)-\varphi\left(x_{i t}^{\text {back }}, t\right)-\phi^{\text {front }}+\phi^{\text {back }}}{R_{\text {ext }}^{P}}
$$

Thus the position independent total internal cell current $j_{n}(x, t)+j_{p}(x, t)=\operatorname{Const}(t)$ can be specified:

1.b $j_{n}\left(x_{i t}^{\text {front }}, t\right)+j_{p}\left(x_{i t}^{\text {front }}, t\right)=j_{\text {int }}(t)$

Furthermore, the electron and hole particle densities at the interface, or the electron/hole currents into the metal contacts can be specified for both contacts.

In the EQ calculation mode, the majority carrier density at the interface under equilibrium $n^{E Q}\left(x_{\text {it }}^{\text {front /back })}\right.$ or $p^{E Q}\left(x_{i t}^{\text {front /back }}\right)$ is given by the majority barrier height $\phi_{B n}^{\text {Schottky }}=q\left\{\phi^{\text {front/back }}-\chi^{\text {front/back }}\right\}, \quad \phi_{B p}^{\text {Schottky }}=q\left\{E_{g}-\phi^{\text {front/back }}+\chi^{\text {front/back }}\right\} \quad$ of $\quad$ the metal/semiconductor contact (with $\chi^{\text {front/back }}$ being the electron affinity of the semiconductor adjacent to the front/ back contact):

2.a, $2 \mathrm{~b} n^{E Q}\left(x_{i t}^{\text {front/back }}\right)=N_{C} e^{-\frac{\phi_{B n}^{\text {Schothy }}}{k T}} \quad$ or $\quad p^{E Q}\left(x_{i t}^{\text {front/back }}\right)=N_{V} e^{-\frac{\phi_{B p}^{\text {Schootly }}}{k T}}$

The corresponding minority carrier density under equilibrium $p^{E Q}\left(x_{\text {it }}^{\text {front } / \text { back }}\right)$ or $n^{E Q}\left(x_{i t}^{\text {front } / \text { back }}\right)$ is then given by the mass action law:

3.a, 3.b $p^{E Q}\left(x_{i t}^{\text {front/back }}\right)=\frac{N_{C} N_{V} e^{-\frac{E_{g}}{k T}}}{n^{E Q}\left(x_{i t}^{\text {front } / \text { back }}\right)} \quad$ or $\quad n^{E Q}\left(x_{i t}^{\text {front/back }}\right)=\frac{N_{C} N_{V} e^{-\frac{E_{g}}{k T}}}{p^{E Q}\left(x_{i t}^{\text {front/back }}\right)}$

As flatband conditions are chosen, the metal work function is calculated to give a zero build in voltage due to the metal/semiconductor contact:

$$
\phi^{\text {front } / \text { back }}=E_{C}-E_{F}\left(x_{i t}^{\text {front } / \text { back }}\right)+\chi^{\text {front/back }}
$$

In all other calculation modes (DC, AC, TR), the electron/hole currents into the metal contact, $j_{n}^{i t, \text { front } / \text { back }}(t), j_{p}^{i t, \text { front } / \text { back }}(t)$ are specified: 
2.a $j_{n}^{i t, \text { front }}(t)=q S_{n}^{\text {front }}\left\{n\left(x_{i t}^{\text {front }}, t\right)-n^{E Q}\left(x_{i t}^{\text {front }}\right)\right\}$

2.b $j_{n}^{i t, \text { back }}(t)=-q S_{n}^{\text {back }}\left\{n\left(x_{i t}^{\text {back }}, t\right)-n^{E Q}\left(x_{i t}^{\text {back }}\right)\right\}$

3.a $j_{p}^{i t, \text { front }}(t)=-q S_{p}^{\text {front }}\left\{p\left(x_{i t}^{\text {front }}, t\right)-p^{E Q}\left(x_{i t}^{\text {front }}\right)\right\}$

3.b $j_{p}^{i t, \text { back }}(t)=q S_{p}^{\text {back }}\left\{p\left(x_{i t}^{\text {back }}, t\right)-p^{E Q}\left(x_{i t}^{\text {back }}\right)\right\}$

Furthermore, if using the DC, AC or TR calculation mode with an external illumination (optical super bandgap generation) enabled, an illumination dependent metal work function is calculated, in order to ensure illumination independent flatband conditions: Assuming a zero internal current density (no netto current through the semiconductor stack), the metal work function is now iteratively calculated from the majority quasi Fermi energy $E_{F n}\left(x_{i t}^{\text {front }}, t\right)$ or $E_{F p}\left(x_{i t}^{\text {front }}, t\right)$ instead from the Fermi energy, in order to ensure a zero build in voltage due to the metal/semiconductor contact

$$
\begin{gathered}
\phi^{\text {front/back }}(t)=E_{C}-E_{F n}\left(x_{i t}^{\text {front/back }}, t\right)+\chi^{\text {front/back }} \quad \text { or } \\
\phi^{\text {front/back }}(t)=E_{C}-E_{F p}\left(x_{i t}^{\text {front/back }}, t\right)+\chi^{\text {front/back }}
\end{gathered}
$$

\subsubsection{Boundary model: Schottky metal/semiconductor contact}

This boundary model can describe metal/semiconductor contacts, which drive the semiconductor into depletion or into accumulation (Sze \& Kwok, 2007). Explicit values of the metal work function $\phi^{\text {front /back }}$ can be specified in order to fix the majority barrier height of the metal/semiconductor contact $\phi_{B n}^{\text {Schottky }}=q\left\{\phi^{\text {front/back }}-\chi^{\text {front/back }}\right\}$, $\phi_{B p}^{\text {Schottky }}=q\left\{E_{g}-\phi^{\text {front/back }}+\chi^{\text {front/back }}\right\}$. Otherwise, this boundary model is totally equivalent to the flatband metal/semiconductor boundary model described above.

\subsubsection{Boundary model: insulator contact}

If the boundary of the semiconductor stack is considered to be insulating, additional interface states can be defined, as according to (Kronik \& Shapira, 1999). They are treated equivalent to the bulk, but with densities given in $\mathrm{cm}^{-2}$ instead of $\mathrm{cm}^{-3}$. For an insulator/semiconductor contact at the front the three boundary conditions are:

1. $\quad 0=-\left.\varepsilon_{0} \varepsilon_{r} \frac{\partial \varphi(x, t)}{\partial x}\right|_{x_{\text {front }}}-q \sum_{\text {trap }} \rho^{\text {trap }}$

2. $0=j_{n}\left(x_{\text {front }}\right)-R_{i t, n}^{\text {front }}$

3. $0=-j_{p}\left(x_{\text {front }}\right)-R_{i t, p}^{\text {front }}$

\subsubsection{Boundary model: metal/insulator/semiconductor contact}

In case of using a metal/insulator/semiconductor MIS contact, the insulator capacity $\mathrm{C}$ has to be additionally specified. At the insulator/semiconductor interface additional interface 
defects can be defined, which are treated equivalent to the bulk but with densities given in $\mathrm{cm}^{-2}$ instead of $\mathrm{cm}^{-3}$, as according to (Kronik \& Shapira, 1999). Depending on whether the MIS contact is defined on only one or on both boundaries two different cases have to be discussed. If both boundaries have an MIS contact, the capacities $C^{\text {front }}, C^{\text {back }}$ of the front and back boundaries can be defined separately. Furthermore, one has to define the voltage fraction $f$ that drops at the front MIS contact compared to the fraction that drops at the back MIS contact. For a given external voltage this defines how the different metal layers are charged. Time dependent boundary conditions (AC or TR calculation mode) for the MIS contact on are not implemented at the current state of AFORS-HET development.

Both semiconductor potentials $\varphi\left(x_{\text {front }}\right)$ and $\varphi\left(x_{\text {back }}\right)$ at the front/back boundary of the semiconductor stack and the metal work functions $\phi^{\text {front } / \text { back }}$ of the front/back contact enter the boundary condition for the electric potential. Also enters the net charge $\rho_{i t}$ of the interface, which has to be calculated by summing over all interface defects. The electron/hole currents into the interface defects $j_{n}\left(x_{\text {front }}\right), j_{p}\left(x_{\text {front }}\right)$, are given by the recombination rates $R_{i t, n}^{\text {front }}, R_{i t, p}^{\text {front }}$ of the interface defects. The three boundary conditions for a MIS contact read:

1.

$$
0=C\left\{V_{\text {ext }}-\left[\varphi\left(x_{\text {back }}\right)-\varphi\left(x_{\text {front }}\right)\right]+\left[\phi^{\text {back }}-\phi^{\text {front }}\right]\right\}-\left.\varepsilon_{0} \varepsilon_{r} \frac{\partial \varphi(x)}{\partial x}\right|_{x_{\text {front }}}-q \sum_{\text {trap }} \rho_{i t}^{\text {trap }}
$$

2.

$$
0=j_{n}\left(x_{\text {front }}\right)-R_{i t, n}^{\text {front }}
$$

3.

$$
0=-j_{p}\left(x_{\text {front }}\right)-R_{i t, p}^{\text {front }}
$$

in the case that only one MIS contact at the front boundary is chosen, and

$$
0=f C^{\text {front }}\left\{V_{\text {ext }}-\left[\varphi\left(x_{\text {back }}\right)-\varphi\left(x_{\text {front }}\right)\right]+\left[\phi^{\text {back }}-\phi^{\text {front }}\right]\right\}-\left.\varepsilon_{0} \varepsilon_{r} \frac{\partial \varphi(x)}{\partial x}\right|_{x_{\text {front }}}-q \sum_{\text {trap }} \rho_{\text {it }}^{\text {trap }}
$$

$$
0=j_{n}\left(x_{\text {front }}\right)-R_{i t, n}^{\text {front }}
$$

$$
0=-j_{p}\left(x_{\text {front }}\right)-R_{i t, p}^{\text {front }}
$$

$$
0=(1-f) C^{\text {back }}\left\{V_{\text {ext }}-\left[\varphi\left(x_{\text {back }}\right)-\varphi\left(x_{\text {front }}\right)\right]+\left[\phi^{\text {back }}-\phi^{\text {front }}\right]\right\}-\left.\varepsilon_{0} \varepsilon_{r} \frac{\partial \varphi(x)}{\partial x}\right|_{x_{\text {back }}}-q \sum_{\text {trap }} \rho_{\text {it }}^{\text {trap }}
$$

$$
0=j_{n}\left(x_{\text {back }}, t\right)-R_{i t, n}^{\text {back }}
$$

in case that two MIS contacts at both boundaries are chosen.

\section{Characterization methods simulated by AFORS-HET}

In the following it is described how the most common solar cell characterization methods are simulated within AFORS-HET, i.e. current-voltage (IV), quantum efficiency (QE), quasisteady-state photoconductance (QSSPC), impedance (IMP, ADM, C-V, C-T, C-f), surface photovoltage (ID-SPV, VD-SPV, WD-SPV) and photo-electro-luminescence (PEL).

\subsection{Measurement model: current-voltage characteristic (IV)}

This measurement varies the external voltage at the boundaries and plots the resulting external current through the semiconductor stack in order to obtain the current-voltage 
characteristic of the simulated structure. For each voltage value the total current through the structure (the sum of the electron and hole current at a boundary gridpoint) is calculated. This can be done in the dark or under an illumination. The measurement model can iterate the specific data points maximum-power point (mpp), open-circuit voltage (Voc), shortcircuit current (Isc) and thus calculate the fill-factor $F F$ and the efficiency Eff of the solar cell, whereas the illumination power density $P_{\text {illumination }}$ in $W / \mathrm{cm}^{2}$ is calculated from the incident photon spectrum:

$$
F F=\frac{V_{m p p} I_{m p p}}{V_{o c} I_{s c}}, \quad E f f=\frac{V_{m p p} I_{m p p}}{P_{\text {illumination }}}=\frac{F F V_{o c} I_{s c}}{P_{\text {illumination }}}
$$

\subsection{Measurement model: quantum efficiency (QE)}

In order to simulate quantum efficiencies, the semiconductor stack is additionally illuminated with a monochromatic irradiation at a certain wavelength $\lambda$, and the difference $\Delta I_{S C}^{i r r a d}$ of the resulting short circuit current with and without the additional irradiation is computed. A quantum efficiency $Q E(\lambda)$ can then defined as

$$
Q E(\lambda)=\frac{\text { number.of.electrons.in.the.external.circuit }}{\text { number.of.photons }}=\frac{\Delta I_{S C}^{\text {irrad }} / q}{\text { number.of.photons }}
$$

Different quantum efficiencies are calculated, depending on the number of photons which are considered: (1) external quantum efficiency (EQE): all photons of the additional irradiation, which are incident on the semiconductor stack, whether they are reflected, absorbed or transmitted, are counted. (2) internal quantum efficiency (IQE): only the absorbed photons of the additional irradiation are counted. Note, that like in a real measurement, photons which are absorbed in the contacts are also counted, despite the fact that they do not contribute to the current. (3) corrected internal quantum efficiency (IQE1): only the photons of the additional irradiation which are absorbed in the semiconductor stack are counted.

\subsection{Measurement model: quasi steady state photoconductance (QSSPC)}

The excess carrier density dependant lifetimes $\tau_{n_{-} \text {all }}(\Delta n), \tau_{p_{-} \text {all }}(\Delta p)$ for a semiconductor stack of the thickness $L$ under a given external illumination are calculated according to the following equations:

$$
\begin{gathered}
\tau_{n_{-} \text {all }}(\Delta n)=\frac{\Delta n}{\Delta G} \quad \tau_{p_{-} \text {all }}(\Delta p)=\frac{\Delta p}{\Delta G} \\
\Delta n=\left(\int d x\left\{n_{\text {illuminated }}(x)\right\}-\int d x\left\{n_{\text {dark }}(x)\right\}\right) / L \\
\Delta p=\left(\int d x\left\{p_{\text {illuminated }}(x)\right\}-\int d x\left\{p_{\text {dark }}(x)\right\}\right) / L \\
\Delta G=\left(\int d x\left\{G_{\text {illuminated }}(x)\right\}-\int d x\left\{G_{\text {dark }}(x)\right\}\right) / L
\end{gathered}
$$


The average dark and illuminated carrier densities and the average generation rate are calculated by integrating over the whole structure. Thus the excess carrier densities $\Delta n, \Delta p$ and the corresponding change in generation rate $\Delta G$ can be calculated. Within the measurement model, the external illumination intensity is varied and the resulting excess carrier dependant lifetimes $\tau_{n_{-} \text {all }}(\Delta n)$ and $\tau_{p_{-} \text {all }}(\Delta p)$ are plotted.

For typical structures that have a c-Si layer with low mobility passivation layers at the front and back additionally c-Si carrier lifetimes $\tau_{n_{-} c-S i}$ and $\tau_{p_{-} c-S i}$ are calculated by only integrating over the c-Si layer. To model the typical QSSPC measurements of passivated c-Si wafers done with the commercially available setup by Sinton Consulting (Sinton \& Cuevas, 1996), an effectively measured carrier lifetime $\tau_{q s s}$ is calculated by the following equation:

$$
\tau_{q s s}=\frac{\Delta n_{c-S i} \mu_{n_{-} c-S i}+\Delta p_{c-S i} \mu_{p_{-} c-S i}}{\mu_{n_{-} c-S i}+\mu_{p_{-} c-S i}} / \Delta G_{c-S i}
$$

\subsection{Measurement model: impedance, capacitance (IMP, ADM, C-V, C-T)}

Both boundaries must be described by a voltage controlled metal-semiconductor contact. Additional to the time independent external DC voltage $V_{e x t}^{D C}$ an alternating sinusoidal AC voltage is superimposed, $\tilde{V}_{\text {ext }}(x, t)=V_{\text {ext }}^{D C}(x)+V_{\text {ext }}^{A C}(x) e^{i \omega t}$, with a small amplitude $V_{e x t}^{A C}$ and a given frequency $f, \omega=2 \pi f$. The resulting external current through the semiconductor stack in the limes of a sufficiency small amplitude is calculated, $\tilde{I}_{\text {ext }}(x, t)=I_{\text {ext }}^{D C}(x)+I_{\text {ext }}^{A C}(x) e^{i \omega(t+\delta)}=I_{\text {ext }}^{D C}(x)+\tilde{I}_{\text {ext }}^{A C}(x) e^{i \omega t}$. It is also sinusoidal and of the same frequency $f$, with an AC-amplitude $I_{e x t}^{A C}$ and a phase shift $\delta$, or with a complex amplitude $\tilde{I}_{\text {ext }}^{A C}$ respectively.

The impedance is defined to be the complex resistance of the semiconductor stack, i.e. the quotient of ac-voltage to ac-current. The admittance is defined to be the complex conductivity of the semiconductor stack, i.e. the quotient of ac-current to ac-voltage. It can be equivalently represented by a parallel circuit of a conductance $G$ and a capacitance $C$.

$$
I M \tilde{P}=\frac{V_{e x t}^{A C}}{\tilde{I}_{e x t}^{A C}}, \quad A D \tilde{M}=\frac{\tilde{I}_{e x t}^{A C}}{V_{e x t}^{A C}}=G+\mathrm{i} 2 \pi f C
$$

Depending on the measurement chosen, the frequency is varied and the amplitude and phase shift of the impedance is plotted (measurement IMP), or the capacitance, conductance and conductance divided by frequency is plotted (measurement ADM). Furthermore, for a fixed frequency $f$, the capacitance can be plotted as a function of the external DC-voltage (measurement $\mathrm{C}-\mathrm{V}$ ) or as a function of the temperature (measurement $\mathrm{C}-\mathrm{T}$ ).

\subsection{Measurement model: surface photovoltage (ID-SPV, VD-SPV, WD-SPV)}

In order to simulate a steady-state surface photovoltage (SPV) signal (Kronik \& Shapira, 1999), the front side boundary should usually be a metal-insulator-semiconductor contact. The semiconductor stack is additionally illuminated with a monochromatic irradiation at a certain wavelength and intensity. The potential difference $\Delta f$ with and without 
monochromatic illumination at the front (first grid point) and at the back (last grid point) of the stack is computed and as output the SPV signal $V_{S P V_{-} \text {front/back }}$ is calculated.

$$
V_{\text {front } / \text { back }}^{S P V}=\Delta \varphi_{\text {front/back }}=\left(\varphi_{\text {front/back }}^{\text {illuminated }}-\varphi_{\text {front/back }}^{\text {dark }}\right)
$$

Note that only one quantity $\varphi_{\text {front }}$ or $\varphi_{\text {back }}$ will change upon illumination, as the potential is fixed to $\varphi=0$ either at the front side or at the back side. Depending on the measurement, either the intensity of the monochromatic illumination is varied (ID-SPV, intensity dependant surface photovoltage), or the external voltage is varied (VD-SPV, voltage dependant surface photovoltage), or the wavelength of the monochromatic illumination is varied (WD-SPV, wavelength dependant surface photovoltage).

\subsection{Measurement model: photo electro luminescence (PEL)}

When an external illumination and/or an external voltage are applied the emitted radiation can be calculated according to the generalized Plank equation (Würfel, 1982).

$$
I(\lambda)=2 c \int d x\left\{\frac{\alpha(\lambda, x)}{\lambda^{5}} \cdot \frac{1}{\exp \left(\left(\frac{h c}{\lambda}-\left(E_{F n}(x)-E_{F p}(x)\right)\right) / k T\right)-1}\right\}
$$

By integration over the whole structure the wavelength dependant emitted intensity to the front and back is calculated taking photon re-absorption into account. For a given absorption coefficient $\alpha$ and a given wavelength $\lambda$ the spectra $I(\lambda)$ of the emitted photons is determined by the splitting of the quasi-Fermi levels of electrons and holes $E_{F n}, E_{F p}$. The external working conditions like external illumination and/or applied voltage that cause the quasi-Fermi level splitting have to be specified. Furthermore the wavelength region for which the emitted intensity is calculated can be selected.

\section{Selected examples on AFORS-HET simulations}

To illustrate the concepts of numerical solar cell simulation, some selected examples simulating a simple amorphous/crystalline silicon solar cell are shown. The absorber of the solar cell (designed for photon absorption) is constituted by a $300 \mu \mathrm{m}$ thick p-doped textured silicon wafer, c-Si, whereas the emitter of the solar cell (designed for minority carrier extraction, that is electron extraction) consists of an ultra thin $10 \mathrm{~nm}$ layer of n-doped, hydrogenated amorphous Silicon, a-Si:H, see Fig. 4. In order to support the lateral electron transport, a transparent conductive oxide layer, TCO, is used as a front side contact. For the sake of simplicity, majority carrier extraction that is hole extraction, is realized as a simple metallic flatband contact to the p-type absorber. Please note, that this solar cell structure is not a high efficiency structure, as a back surface field region, BSF, for hole extraction in order to avoid contact recombination, has not been used. However, this structure has been chosen, as it clearly reveals the properties of an amorphous/crystalline heterojunction interface.

This interface is crucial for the performance of an amorphous/crystalline heterojunction solar cell: By an adequate wet-chemical pre-treatment of the wafer prior to the deposition of 
a-Si:H onto the surface of the silicon wafer, one has to ensure that an a-Si:H/c-Si heterocontact with a low a-Si:H/c-Si interface state density, $D_{i t}$, will form. The influence of $D_{i t}$ on the solar cell performance as well as on various solar cell characterisation methods will be shown. Thus a sensitivity analysis of different measurement methods in order to measure an unknown $D_{i t}$ is performed my means of numerical simulation.

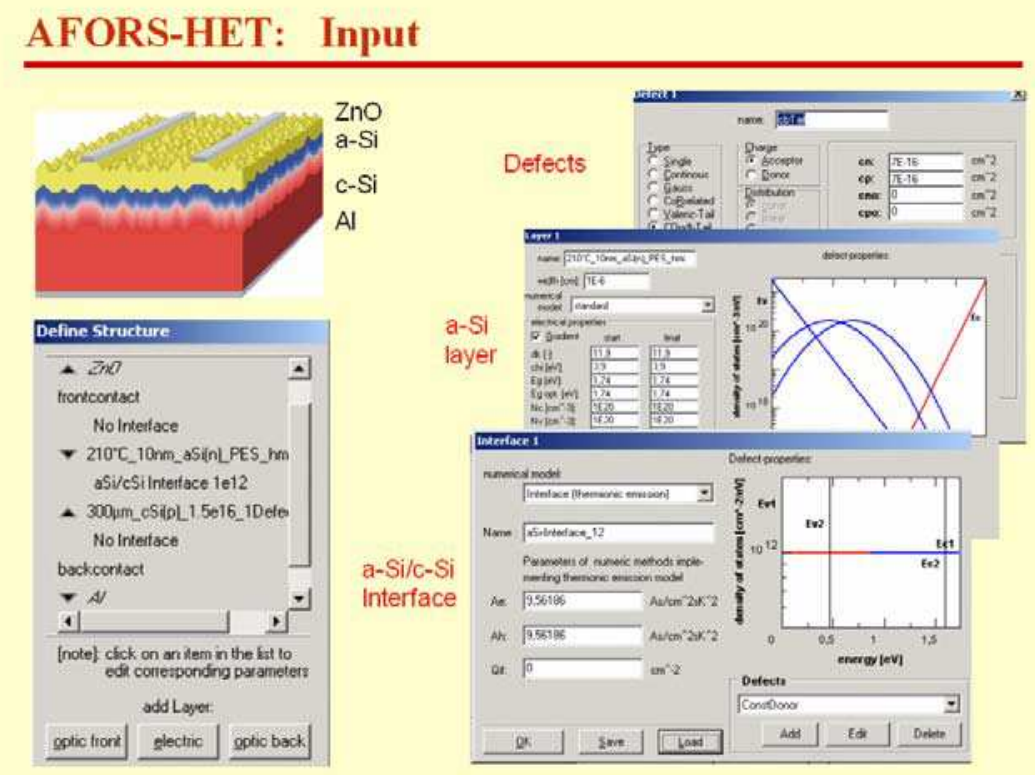

Fig. 4. Screenshots of typical AFORS-HET input: Simulation of TCO/a-Si:H(n)/c-Si(p)/Al heterojunction solar cells. (left) layer sequence, (right) defect distributions $N_{\text {trap }}(E)$ of the a-Si:H(n) layer and of the a-Si:H(n)/c-Si(p) interface.

Fig. 4 shows typical screenshots of an AFORS-HET input while modelling the above mentioned TCO/a-Si:H(n)/c-Si(p)/Al heterojunction solar cell. In order to model the c-Si absorber, the bulk model "crystalline silicon" is chosen, specifying the appropriate doping (i.e. $N_{A}=1.510^{16} \mathrm{~cm}^{-3}$ ) and the appropriate lifetime of the wafer (i.e. specifying a defect density of a single midgap defect $N_{t}=1.10^{10} \mathrm{~cm}^{-3}$, which corresponds to a mean lifetime of $1 \mathrm{~ms}$ as indicated in the input window). In order to model the a-Si:H emitter, the bulk model "standard semiconductor" is chosen, specifying the measured density of state distributions within the bandgap of a-Si:H (Korte \& Schmidt, 2008), see Fig. 4. I.e. the measured Urbach tail states and the measured dangling bond states of a-Si:H have to be stated and the doping density $N_{D}$ has to be adjusted to a value which leads to the measured Fermi level to valence band distance $E_{F}-E_{V}=250 \mathrm{meV}$ of a-Si:H (Korte \& Schmidt, 2008). Furthermore, the electron affinity of a-Si:H has to be adjusted to a value in order to represent the measured valence band offset $\Delta E_{V}=E_{V}^{c S i}-E_{V}^{a S i}=450 \mathrm{meV}$ (Korte \& Schmidt, 2008). For modeling the a-Si/c-Si interface, the interface model "drift diffusion interface" is chosen, assuming a simple constant distribution of interface defects within the bandgap, exhibiting a donor like 
character below midgap and an acceptor like character above midgap, see Fig.4. The TCO layer at the front is modelled as an optical layer, thus at the front contact the measured TCO absorption (Schmidt et. al., 2007) as well as the measured solar cell reflection due to the surface texturing (Schmidt et. al., 2007) is specified. Therefore, for the optical calculation the optical model "Lambert-Beer absorption" has to be specified.

\subsection{Optical calculation}

Fig. 5 shows the resulting spectral absorptions of the incoming AM 1.5 illumination within the different layers of the solar cell: More than half of the low wavelength radiation $(\lambda \leq 350 \mathrm{~nm})$ is absorbed within the $80 \mathrm{~nm}$ thick TCO layer and is therefore lost for solar energy conversion. Also the defect-rich, ultra-thin a-Si:H emitter is significantly absorbing photons up to $\lambda \leq 600 \mathrm{~nm}$. All photons with $\lambda \leq 600 \mathrm{~nm}$, which are not absorbed, are reflected. Most photons with $\lambda \geq 800 \mathrm{~nm}$, which are not absorbed by the solar cell absorber, are transmitted, some of them are reflected, a few of them are absorbed in the TCO layer due to free carrier absorption. After exceeding the bandgap of the c-Si absorber (for $\lambda \geq 1120 \mathrm{~nm}$ ) there is no more photon absorption in the absorber.

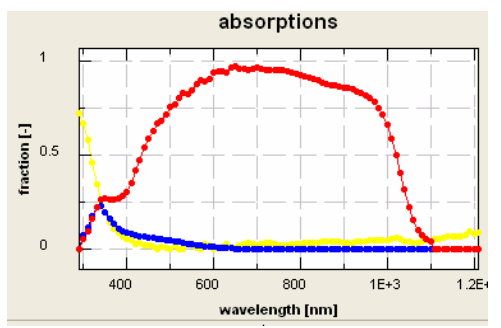

Fig. 5. Screenshot of the spectral absorption within the different solar cell layers (yellow: TCO layer, blue: a-Si:H emitter layer, red: c-Si absorber layer).

\subsection{Equilibrium band diagrams}

Fig. 6 shows the resulting equilibrium band diagrams (conduction band energy, valence band energy and Fermi energy as a function of the position within the solar cell) assuming different interface state densities $D_{i t}$, after an electrical calculation has been performed.
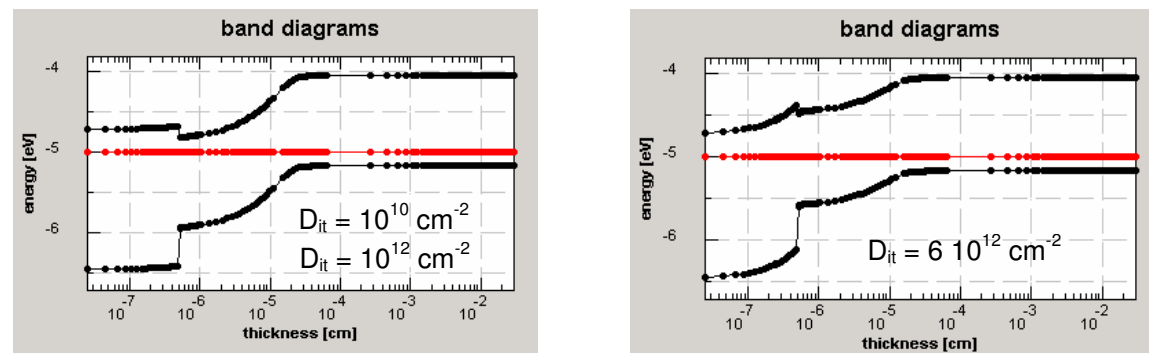

Fig. 6. Screenshots of equilibrium band diagrams (red: Fermi energy, black: valence and conduction band energy) for three different a-Si:H/c-Si interface state densities $D_{i t}$.

Note that the equilibrium band diagram does not change until $D_{i t} \geq 210^{12} \mathrm{~cm}^{-2}$. 


\subsection{Current-voltage characteristics}

However, if one looks at the solar cell performance, i.e. if one calculates the corresponding current-voltage characteristics, $D_{i t}$ will reduce the open-circuit voltage of the solar cell for $D_{i t} \geq 110^{10} \mathrm{~cm}^{-2}$, see Fig. 7. Even if interface states in a comparatively low concentration are formed, i.e. $110^{10} \mathrm{~cm}^{-2} \leq D_{i t} \leq 510^{10} \mathrm{~cm}^{-2}$, this will significantly reduce the solar cell efficiency.

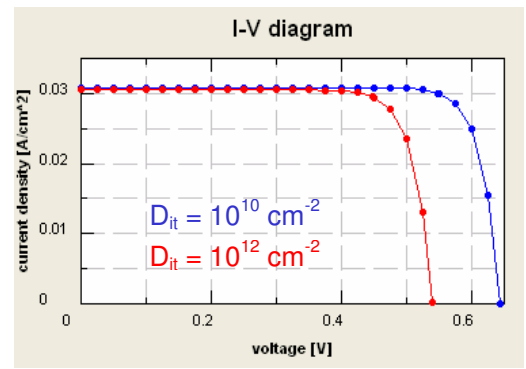

Fig. 7. Screenshot of a current-voltage simulation under AM 1.5 illumination for two different a-Si:H/c-Si interface state densities $D_{i t}$.

\subsection{Quantum efficiency}

The influence of $D_{i t}$ is not noticeable in a quantum efficiency measurement, as the shortcircuit current density is not affected due to a $D_{i t}$ variation, and quantum efficiency is a measure for the excess carrier collection efficiency under short circuit conditions. In Fig. 8 internal as well as external quantum efficiency is shown (IQE, EQE), whereas the difference of the two results from the measured reflection losses.

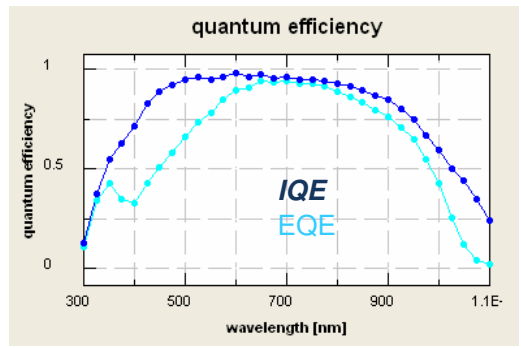

Fig. 8. Screenshot of a quantum efficiency simulation (there is no difference for different a-Si:H/c-Si interface state densities $D_{i t}$ ).

\subsection{Impedance, capacitance}

If one monitors temperature dependent impedance in the dark (i.e. if one calculates the resulting conductance and capacitance as a function of temperature), the onset of the change of the equilibrium band bending due to an increasing $D_{i t}$ can be detected. As soon as the increasing $D_{i t}$ starts to change the equilibrium band bending, an additional peak in the conductance spectra evolves (Gudovskikh et. al., 2006), see Fig. 9. Thus, dark capacitancetemperature (C-T) measurements are sensitive to interface states only for $D_{i t} \geq 2.10^{12} \mathrm{~cm}^{-2}$. 


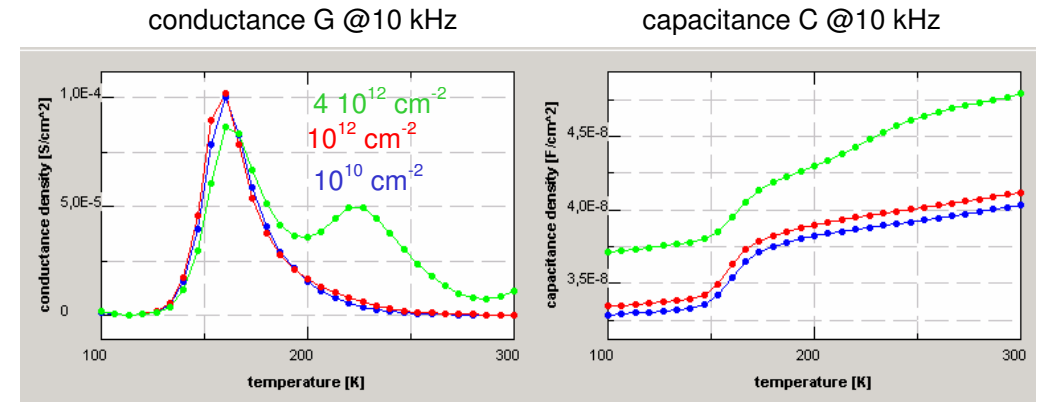

Fig. 9. Screenshot of a capacitance-temperature simulation at an AC frequency of $10 \mathrm{kHz}$ for three different a-Si:H/c-Si interface state densities $D_{i t}$.

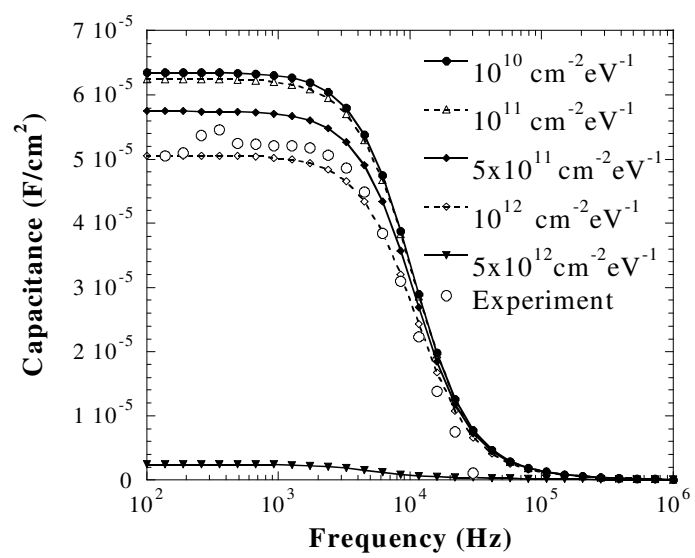

Fig. 10. Comparison of simulated and measured capacitance-frequency measurements under AM1.5 illumination for different a-Si:H/c-Si interface state densities $D_{i t}$. Data from (Gudovskikh et. al., 2006).

In order to enhance the sensitivity towards $D_{i t}$, measurements under illumination have to be performed. Fig. 10 shows an example of an illuminated capacitance-frequency (C-f) measurement, where the corresponding simulations are compared to a real experiment (Gudovskikh et. al., 2006). According to the simulation, the $D_{i t}$ of the solar cell under investigation was in the range $D_{i t} \approx 8.10^{11} \mathrm{~cm}^{-2}$. A sensitivity analysis of this measurement technique indicates a sensitivity towards $D_{i t}$ for $D_{i t} \geq 1.10^{11} \mathrm{~cm}^{-2}$. However, this is still not sufficient in order to characterize well passivated solar cells with a low $D_{i t}$ in the range $110^{10} \mathrm{~cm}^{-2} \leq D_{i t}<110^{11} \mathrm{~cm}^{-2}$.

\subsection{Photoluminescence}

Photoluminescence proofs to be quite sensitive to $D_{i t}$. This is because this measurement performs without current extraction. As an example, Fig. 11 shows the simulated steadystate photoluminescence spectra as well as the transient photoluminescence decay (after an 
integration of the spectra) due to a pulse-like excitation for two different values of $D_{i t}=1.10^{10} \mathrm{~cm}^{-2}$ and $D_{i t}=1.10^{12} \mathrm{~cm}^{-2}$. If one integrates the spectra, the simulated measurement signals differ for more than one order of magnitude.
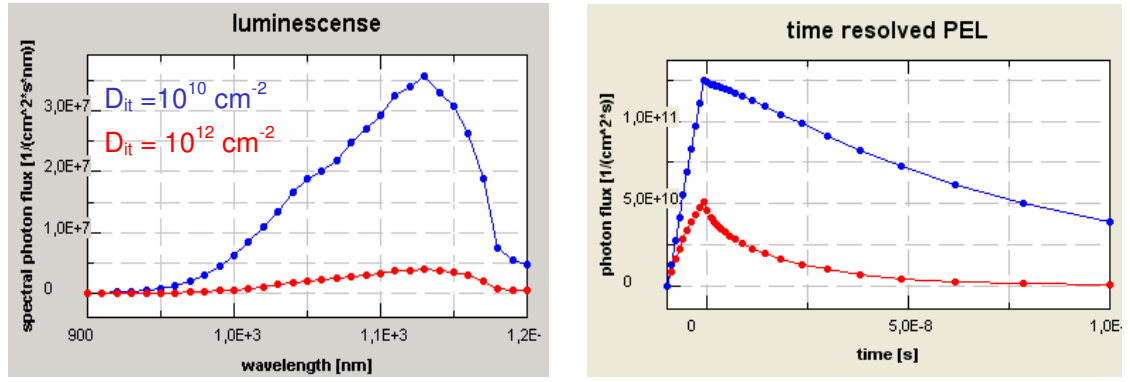

Fig. 11. Screenshots of photoluminescence simulations for two different a-Si:H/c-Si interface state densities $D_{i t}$. (left) steady-state photoluminescence spectra, (right) transient photoluminescence decay after a pulse-like excitation.
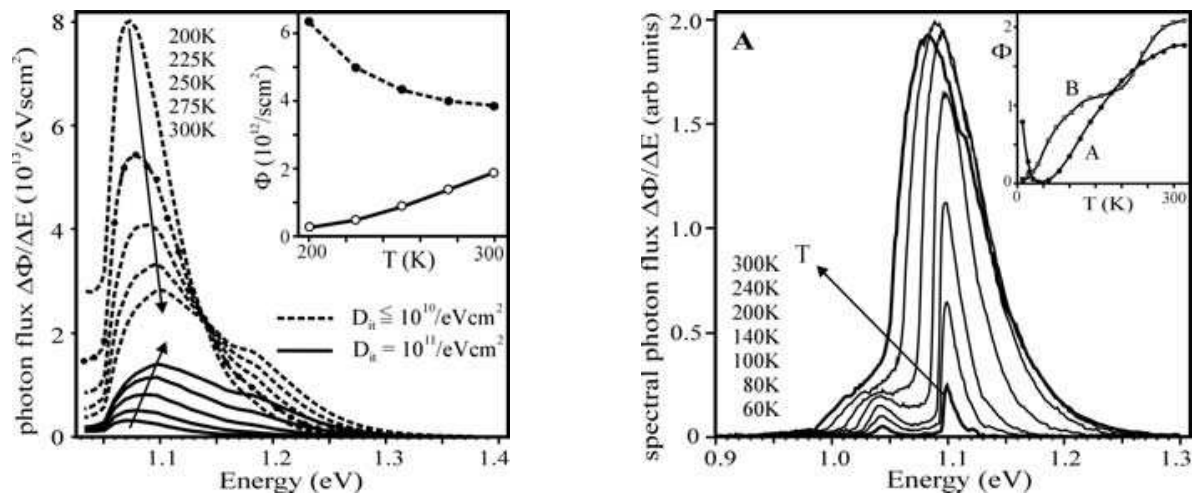

Fig. 12. (left) Simulated temperature dependant photoluminescence measurements for different a-Si:H/c-Si interface state densities $D_{i t}$. (right) Measured temperature dependant photoluminescence. Data from (Fuhs et. al, 2006).

The sensitivity towards $D_{i t}$ can even be more enhanced, if one performs temperature dependant photoluminescence measurements, see Fig. 12. Here the character of the measurement even changes if $D_{i t}$ is in the range $110^{10} \mathrm{~cm}^{-2} \leq D_{i t}<110^{11} \mathrm{~cm}^{-2}$. For $D_{i t} \leq 1.10^{10} \mathrm{~cm}^{-2}$ the spectral emission decreases with increasing temperature, see Fig. 12, thus indicating a non noticeable amount of interface defects, whereas for example for $D_{i t}=1.10^{11} \mathrm{~cm}^{-2}$ an increasing spectral emission with increasing temperature is observed (Fuhs et. al, 2006).

\section{Conclusion}

A mathematical description of AFORS-HET, version 2.4, a one dimensional computer program for the simulation of solar cells and solar cell characterization methods has been 
stated. Some selected examples, simulating amorphous/crystalline silicon heterojunction solar cells and investigating the sensitivity of various measurement methods towards the interface state density $D_{i t}$, were presented.

\section{References}

Anderson, R. L. (1962). Experiments on Ge-GaAs Heterojunctions, Solid State Electron., 5 (1962), 341-51

Basore, P.; Clugston, D. A. (1997). PC1D, Version 5.9, Copyright 2003, University of New South Wales, latest publication describing the program: PC1D Version 5: 32-bit Solar Cell Simulation on Personal Computers, 26th IEEE Photovoltaic Specialists Conf. (Sept 1997)

Fuhs, W.; Laades, L.; v.Maydell, K.; Stangl, R.; Gusev, O.B.; Terukov, E.I.; KazitsynaBaranovski, S.; Weiser, G. (2006). Band-edge electroluminescence from amorphous/crystalline silicon heterostructure solar cells, Journal of Non-Crystalline Solids, 352 (2006) 1884-1887

Gudovskikh, A.S.; Kleider, J.P.; Stangl, R. (2006). New approach to capacitance spectroscopy for interface characterization of a-Si:H/c-Si heterojunctions, Journal of NonCrystalline Solids, 352 (2006) 1213-1216

Korte, L.; Schmidt, M. (2008). Investigation of gap states in phosphorous-doped ultra-thin a-Si:H by near-UV photoelectron spectroscopy, in: J. Non. Cryst. Sol. 354 (2008) 2138-2143

Kronik, L.; Shapira, Y. (1999). Surface Photovoltage Phenomena: Theory, Experiment, and Applications, Surface Science Reports, 37 (1999), 5-206

Kundert et. al. (1988). A sparse linear equation solver, department of electrical engineering and computer science, Berkeley, CA, USA, 1988, available from: http://wwwrab.larc.nasa.gov/nmp/nmpCode.htm

Sah, C.; Shockley, W. (1958). Electron-Hole Recombination Statistics in Semiconductors through Flaws with Many Charge Conditions, Physical Review, 109 (1958), 1103

Schmidt, M.; Korte, L.; Laades, A.; Stangl, R.; Schubert, Ch.; Angermann, H.; Conrad, E.; v.Maydell, K. (2007). Physical aspects of a-Si:H/c-Si hetero-junction solar cells, Thin Solid Films 515 (2007), p. 7475-7480

Selberherr, S. (1984). Analysis and simulation of semiconductor devices, Springer Verlag, 1984

Shockley, W.; Read, W. T. (1952). Statistics of the Recombinations of Holes and Electrons, Physical Review, 87 (1952), 835

Sinton, R.A.; Cuevas, A. (1996). Contactless determination of current-voltage characteristics and minority-carrier lifetimes in semiconductors from quasi-steady-state photoconductance data, Applied Physics Letters, 69 (1996), 2510-2512

Sze, S. M.; Kwok, K. N. (2007). Physics of Semiconductor Devices, John Wiley \& Sons, Inc., Hoboken, New Jersey, 2007

Würfel, P. (1982). The chemical potential of radiation, Journal of Physics C, 15 (1982), 39673985 


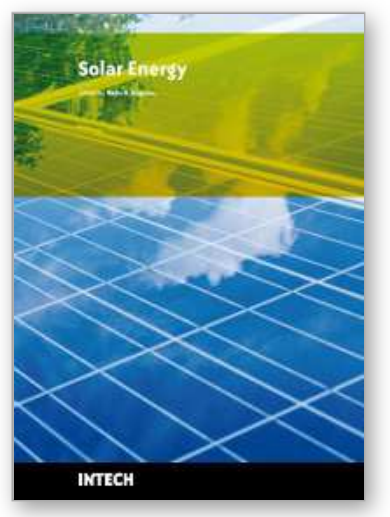

\author{
Solar Energy \\ Edited by Radu D Rugescu
}

ISBN 978-953-307-052-0

Hard cover, 432 pages

Publisher InTech

Published online 01, February, 2010

Published in print edition February, 2010

The present "Solar Energy" science book hopefully opens a series of other first-hand texts in new technologies with practical impact and subsequent interest. They might include the ecological combustion of fossil fuels, space technology in the benefit of local and remote communities, new trends in the development of secure Internet Communications on an interplanetary scale, new breakthroughs in the propulsion technology and others. The editors will be pleased to see that the present book is open to debate and they will wait for the readers' reaction with great interest. Critics and proposals will be equally welcomed.

\title{
How to reference
}

In order to correctly reference this scholarly work, feel free to copy and paste the following:

Rolf Stangl, Caspar Leendertz and Jan Haschke (2010). Numerical Simulation of Solar Cells and Solar Cell Characterization Methods: the Open-Source on Demand Program AFORS-HET, Solar Energy, Radu D Rugescu (Ed.), ISBN: 978-953-307-052-0, InTech, Available from: http://www.intechopen.com/books/solarenergy/numerical-simulation-of-solar-cells-and-solar-cell-characterization-methods-the-open-source-ondeman

\section{INTECH}

open science | open minds

\section{InTech Europe}

University Campus STeP Ri Slavka Krautzeka 83/A 51000 Rijeka, Croatia

Phone: +385 (51) 770447

Fax: +385 (51) 686166

www.intechopen.com

\section{InTech China}

Unit 405, Office Block, Hotel Equatorial Shanghai No.65, Yan An Road (West), Shanghai, 200040, China 中国上海市延安西路65号上海国际贵都大饭店办公楼405单元 Phone: +86-21-62489820

Fax: +86-21-62489821 
(C) 2010 The Author(s). Licensee IntechOpen. This chapter is distributed under the terms of the Creative Commons Attribution-NonCommercialShareAlike-3.0 License, which permits use, distribution and reproduction for non-commercial purposes, provided the original is properly cited and derivative works building on this content are distributed under the same license. 